Alsayednoor, J., Harrison, P. , Dobbie, M., Costantini, R. and Lennard, F. (2019) Evaluating the use of digital image correlation for strain measurement in historic tapestries using representative deformation fields. Strain, 55(2), e12308.

There may be differences between this version and the published version. You are advised to consult the publisher's version if you wish to cite from it.

This is the peer reviewed version of the following article:

Alsayednoor, J., Harrison, P. , Dobbie, M., Costantini, R. and Lennard, F. (2019) Evaluating the use of digital image correlation for strain measurement in historic tapestries using representative deformation fields. Strain, 55(2), e12308. (doi:10.1111/str.12308)

This article may be used for non-commercial purposes in accordance with Wiley Terms and Conditions for Self-Archiving.

http://eprints.gla.ac.uk/176178/

Deposited on: 03 January 2019

Enlighten - Research publications by members of the University of Glasgow http://eprints.gla.ac.uk/ 


\section{Evaluating the Use of Digital Image Correlation for Strain Measurement in Historic Tapestries using Representative Deformation Fields}

\begin{tabular}{|r|l|}
\hline Journal: & Strain \\
\hline Manuscript ID & STRAIN-1433.R1 \\
\hline Danuscript Type: & Special Issue Article \\
\hline Author: & 16 -Oct-2018 \\
\hline & $\begin{array}{l}\text { Alsayednoor, Jafar; University of Glasgow College of Science and } \\
\text { Engineering } \\
\text { Harrison, Philip; University of Glasgow, School of Engineering } \\
\text { Dobbie, Maggie; Glasgow Museums } \\
\text { Costantini, Rosa; University of Glasgow College of Arts } \\
\text { Lennard, Frances; University of Glasgow College of Arts }\end{array}$ \\
\hline Keywords: & $\begin{array}{l}\text { tapestry, digital image correlation, finite element modelling, full-field } \\
\text { monitoring, textile conservation }\end{array}$ \\
\hline &
\end{tabular}




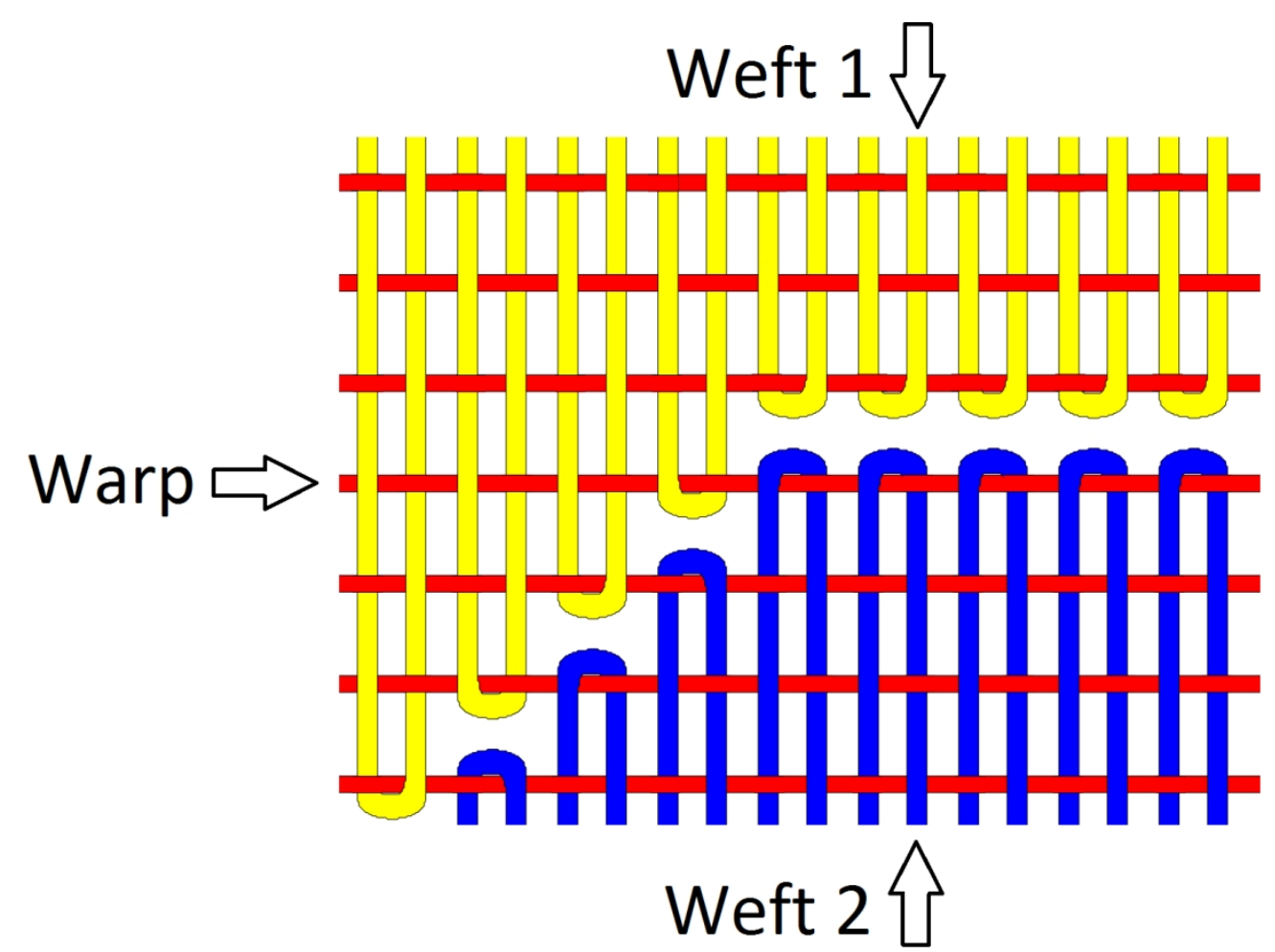

Figure 1. Schematic of the slits occurring between different coloured weft regions of the tapestry 


\section{A. Deform finite element model}

B. Interpolate between nodal displacements

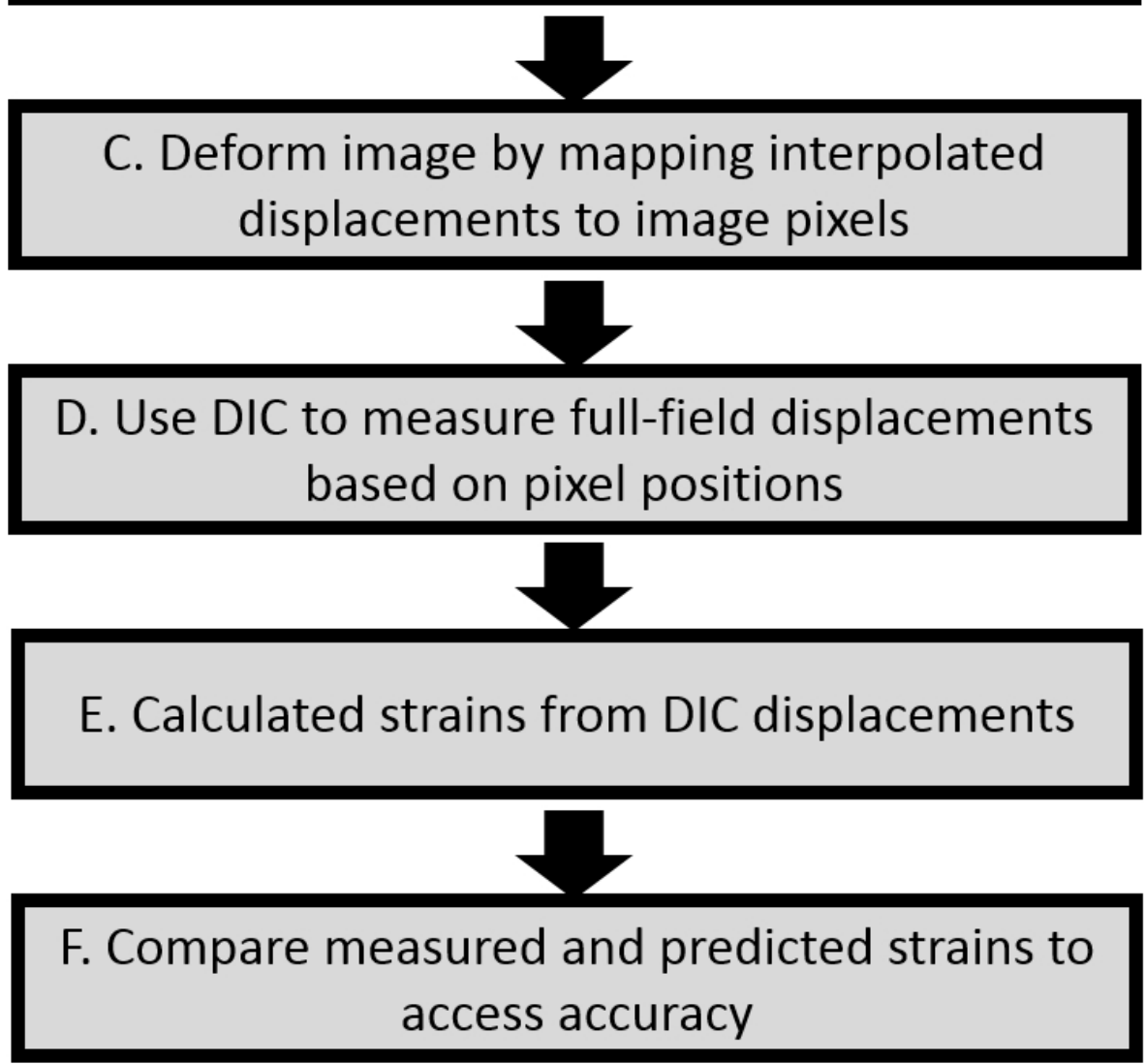

Figure 2. Flow chart showing DIC assessment process. 


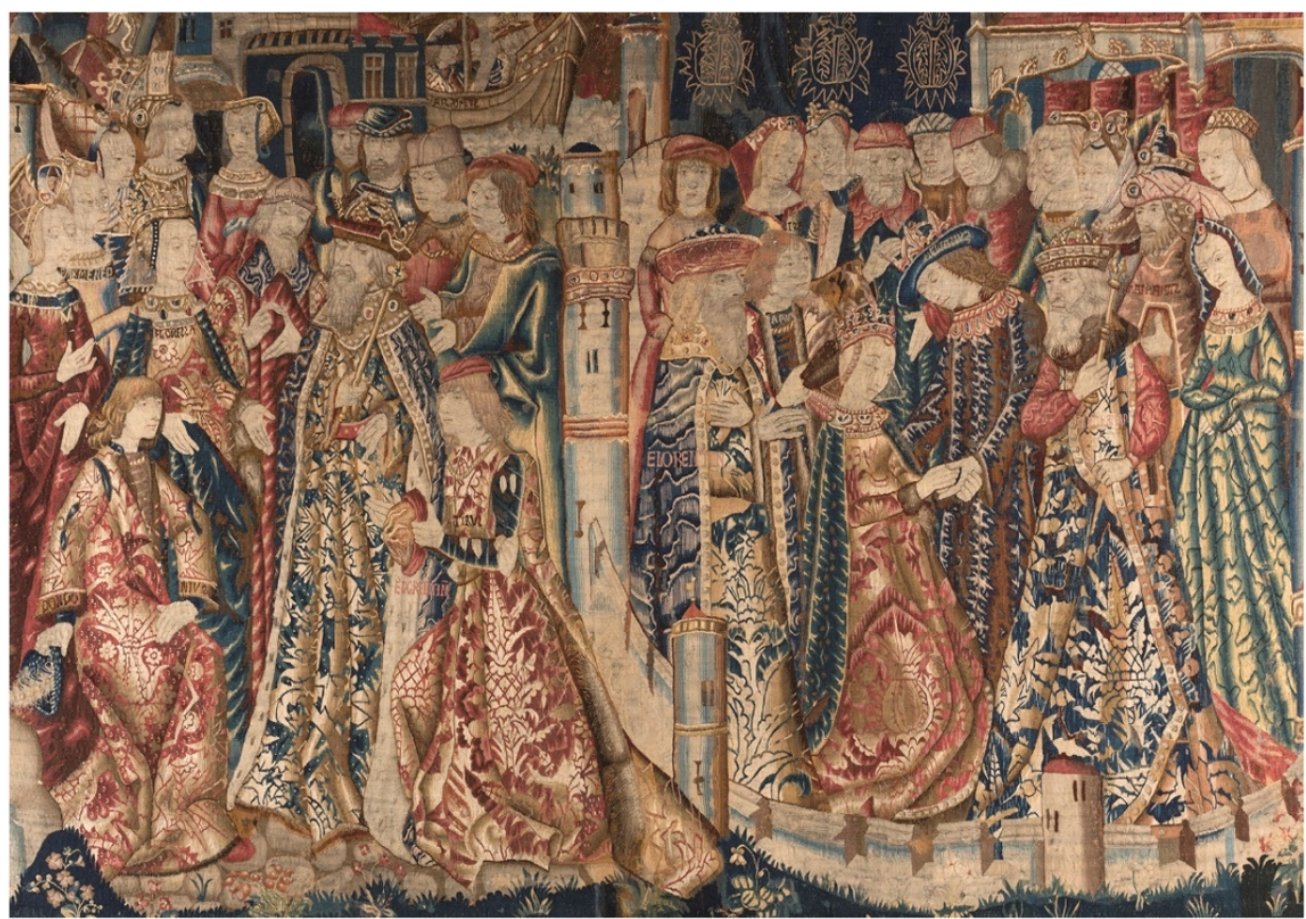

Figure 3. Two Episodes from a Chivalric Romance, Perhaps the Tale of Florence of Rome, c. 1480, $\sim 2.10 \times$ $3.0 \mathrm{~m}$, The Burrell Collection, Glasgow Museums, (C) CSG CIC Glasgow Museums Collection. 


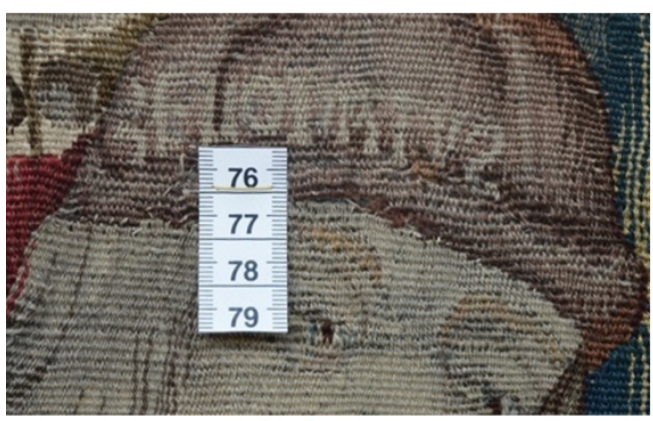

(a)

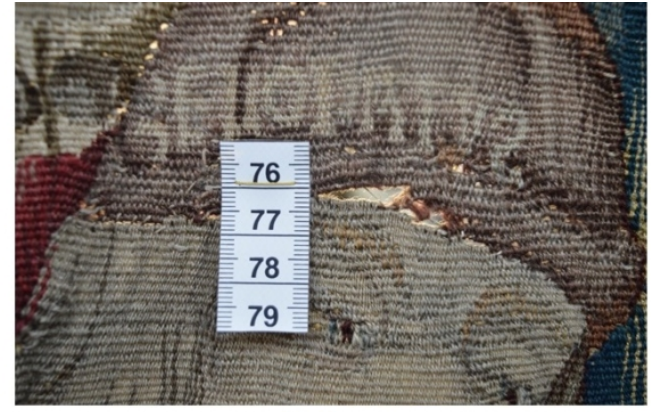

(b)

Figure 4: A close up from Figure 3; the effect of self-weight loading on damage propagation (a) just before and (b) two weeks after unsupported tapestry display. Velcro fastener was used to hang the tapestry 


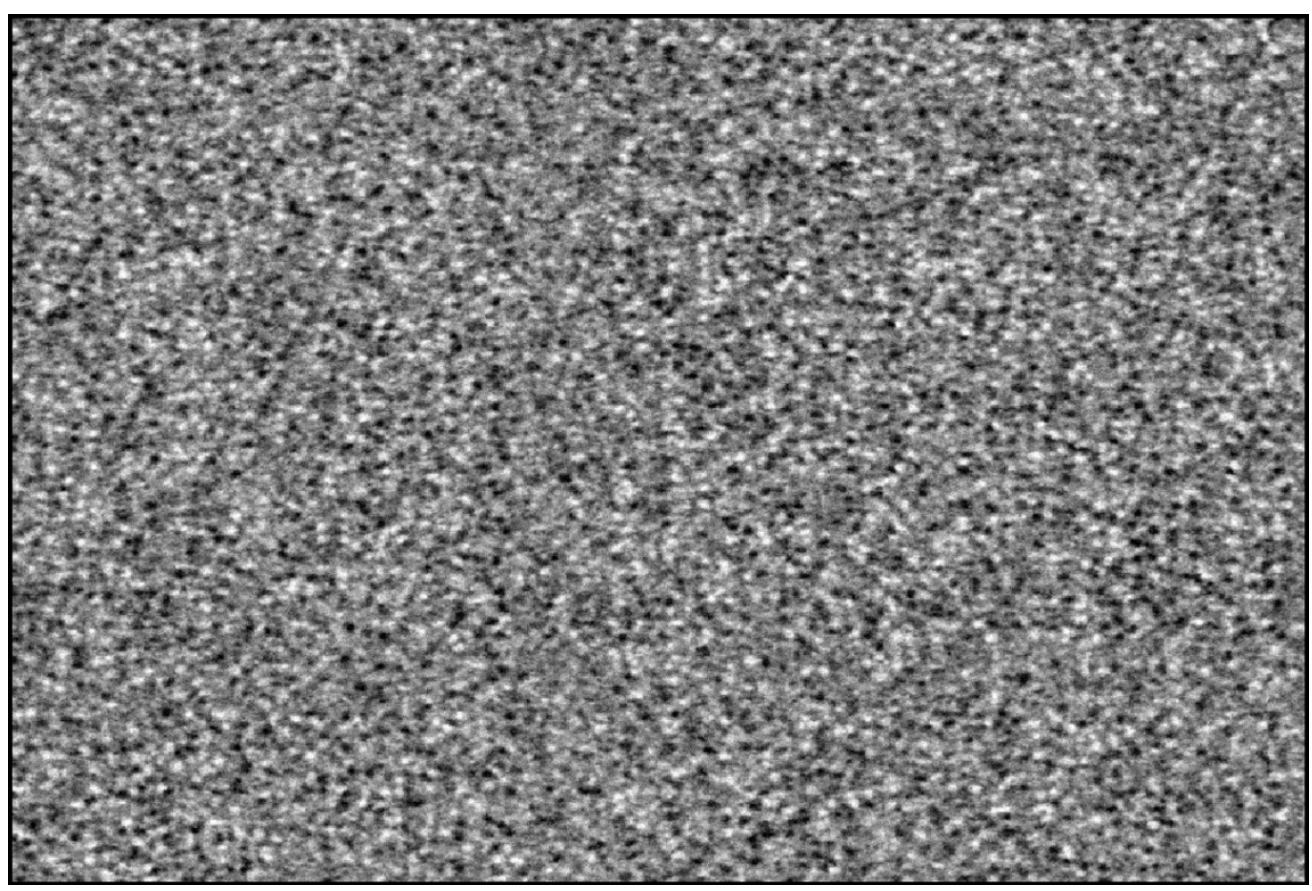

Figure 5: Digitally generated using Perlin speckle pattern with identical dimensions to the corresponding tapestry

$426 \times 284 \mathrm{~mm}(72 \times 72 \mathrm{DPI})$ 


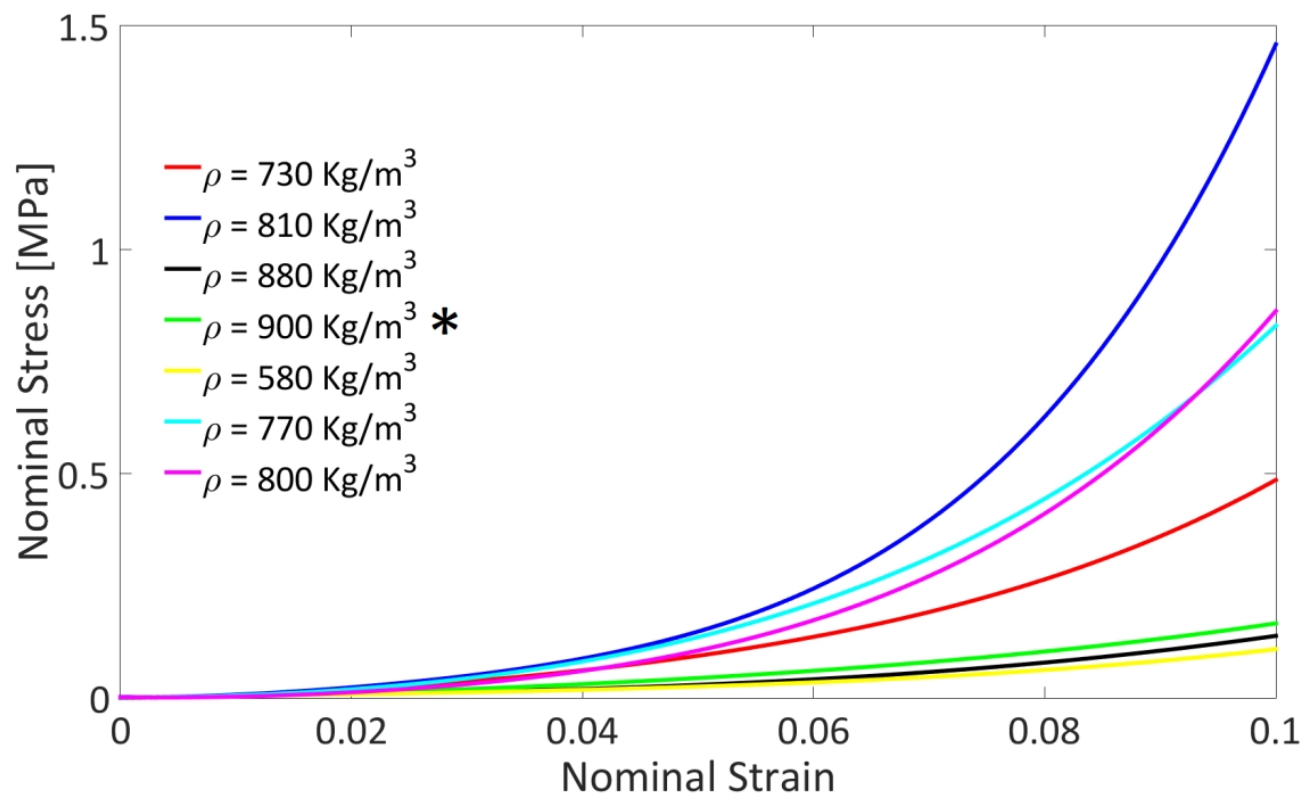

Figure 6: Nominal stress-strain curves for 20 specimens extracted from 7 historic tapestries (different colour code represents different historic tapestry). The selected curve is highlighted by the star symbol. 
(a)
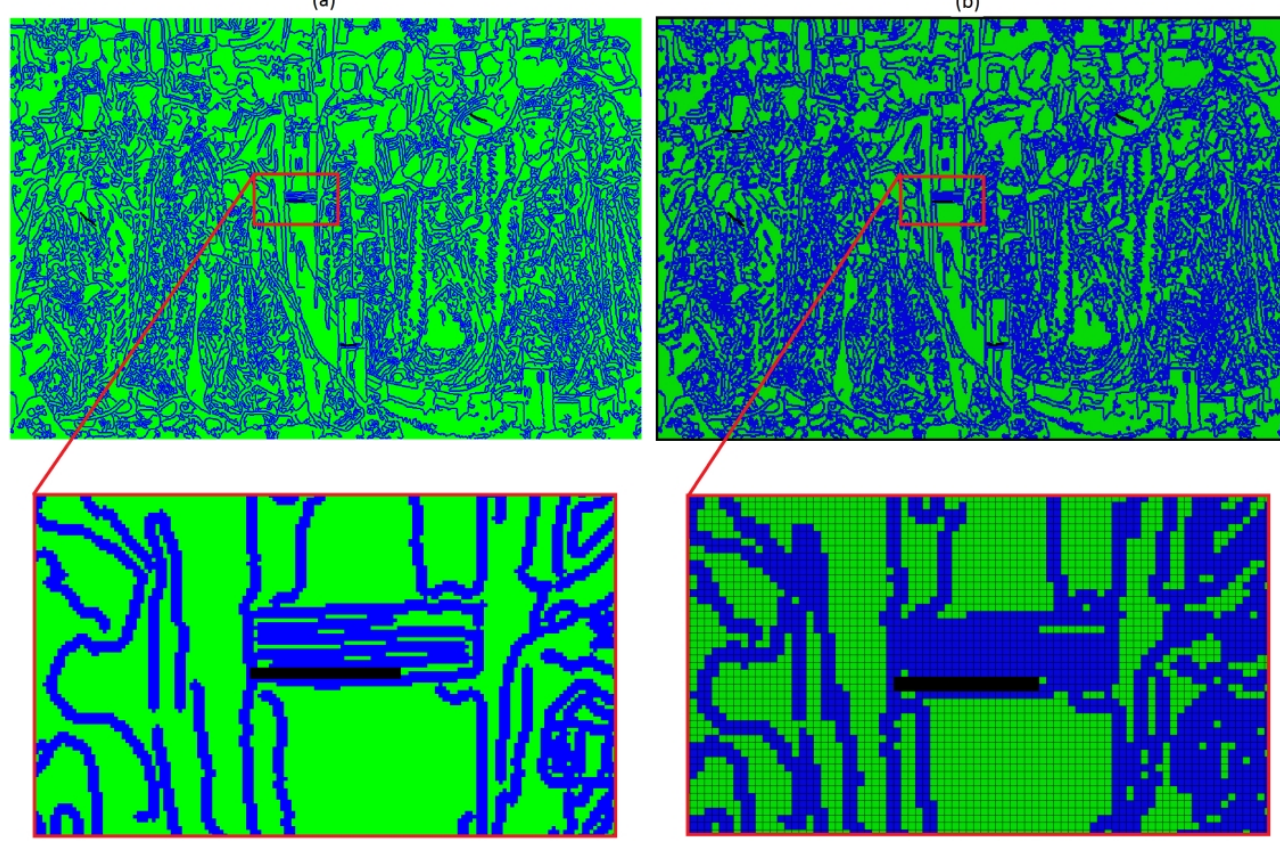

Figure 7: (a) Weak stitched zones, identified using the Canny edge detector (blue colours). Fabric tears were introduced by elimination of the elements along the edges (black colour), (b) the corresponding Abaqus model. 


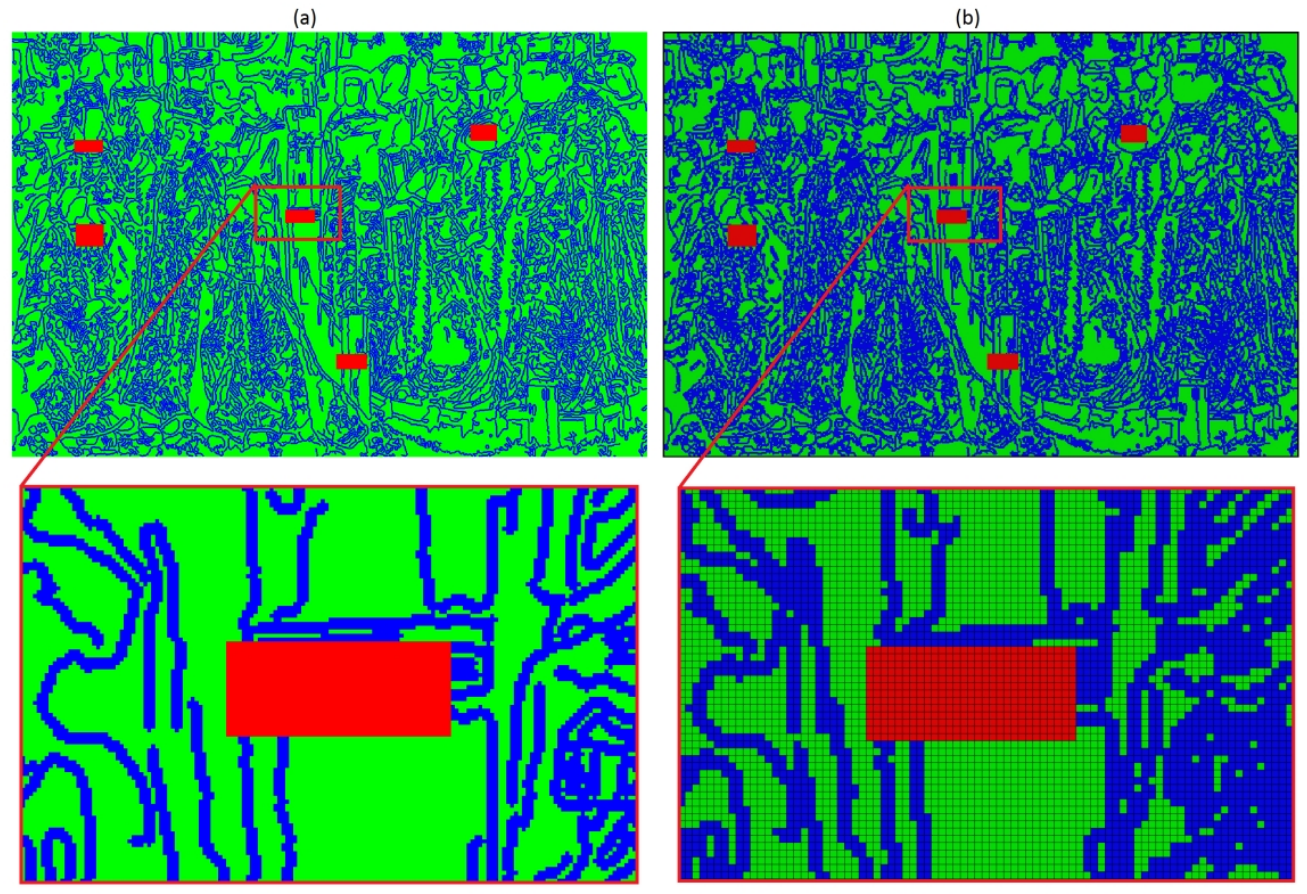

Figure 8: (a) The patch support sections overlaying the tears' regions (red colours), (b) the corresponding Abaqus modelling. 


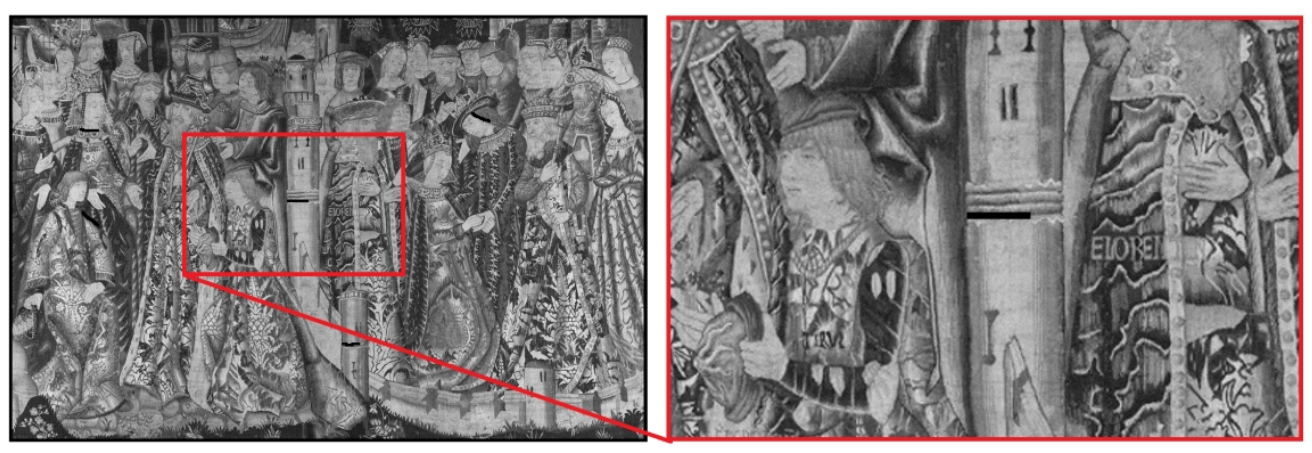

Figure 9: Three times image magnification of the region of interest for the tapestry image containing tears. $873 \times 290 \mathrm{~mm}(72 \times 72 \mathrm{DPI})$ 


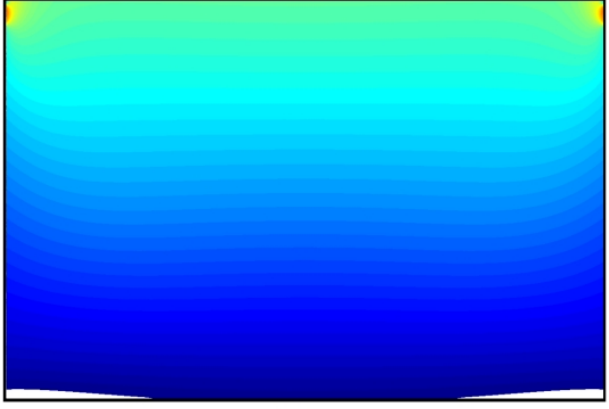

(a)

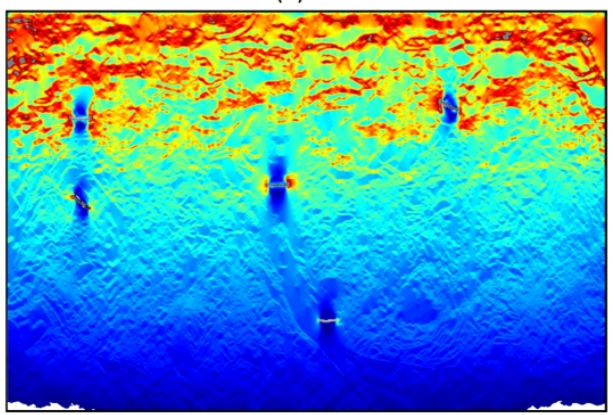

(c)

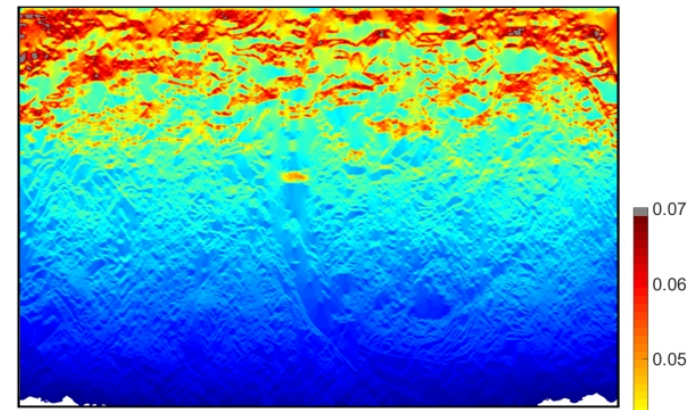

(b)

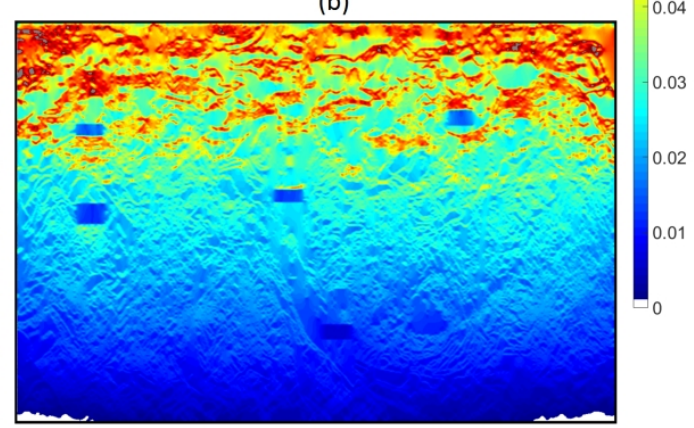

(d)

Figure 10: Simulated vertical strain fields for the $1.5 \times 1.2 \mathrm{~m}$ tapestry: (a) with homogenous material assignment, (b) including stitched join lines, (c) including randomised tears along stitched join lines, (d) including patch restorations over tears 


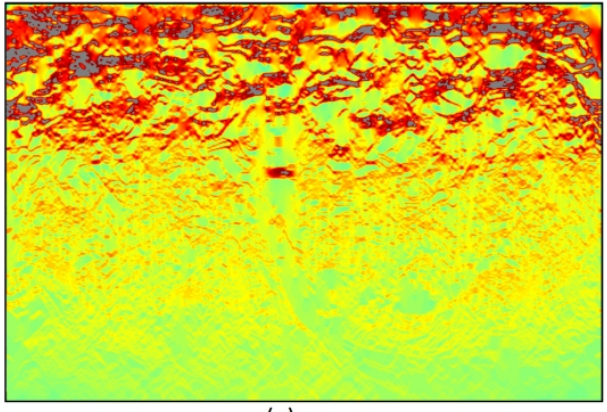

(a)

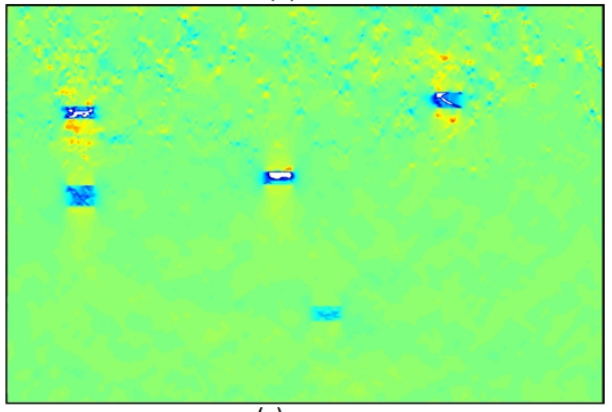

(c)

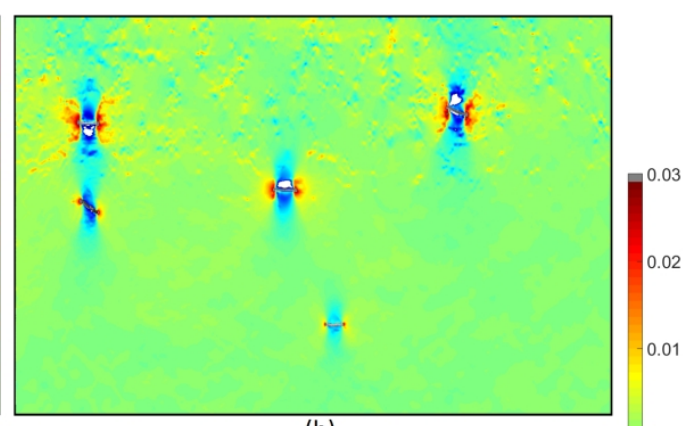

(b)

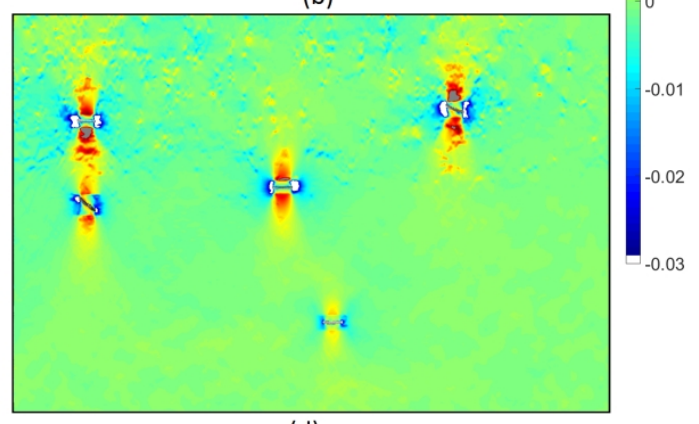

(d)

Figure 11: (a) Homogeneous strain field subtracted from heterogeneous (join line) strain field, (b) heterogeneous strain field subtracted from tear strain field, (c) heterogeneous strain field subtracted from patched strain field, (d) the tear strain field subtracted from the patched strain field. 


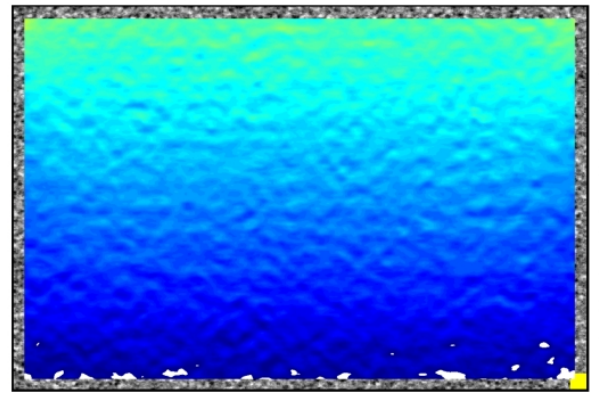

(a)

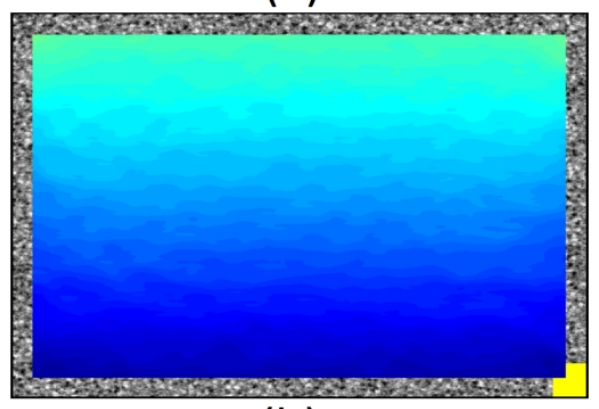

(b)

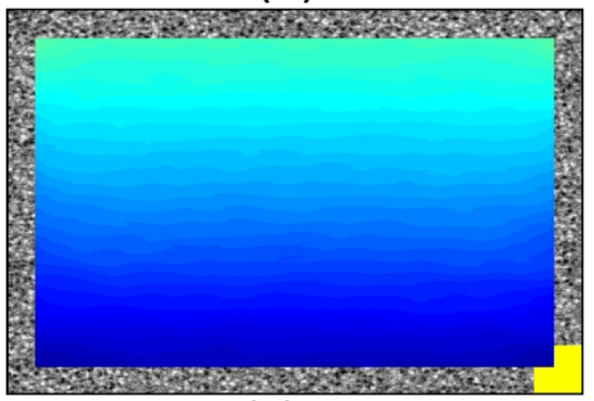

(c)

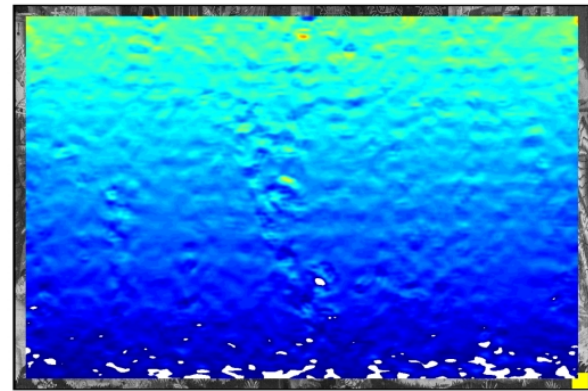

(d)

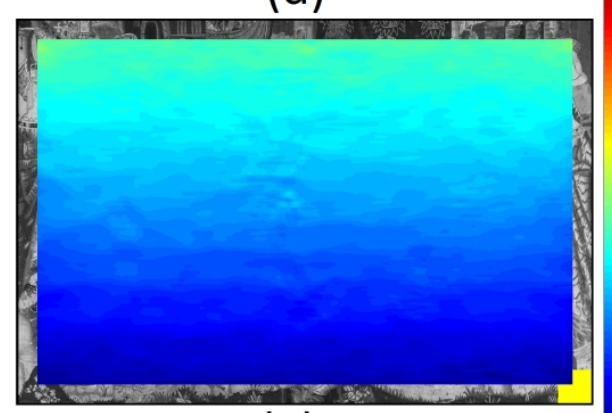

(e)

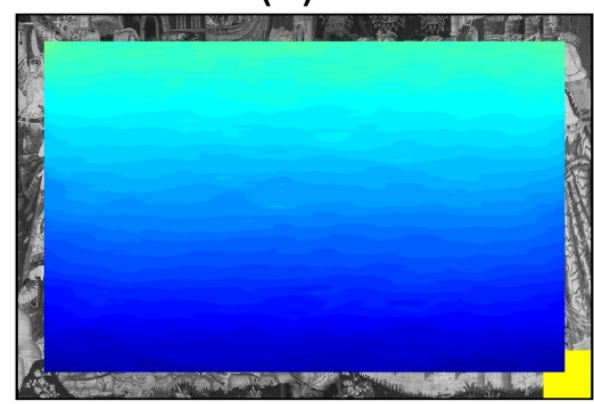

(f)

Figure 12: Left: DIC-measured LE22 strains deformed using predicted simulation strains from homogenous material assignment (Case 1). (a-c) using speckle pattern image to track deformation and subset sizes, shown by yellow corner square, of (a) 33 pixels, (b) 69 pixels, (c) 99 pixels. (d-f) using tapestry image to track deformation and subset sizes of (d) 33 pixels, (e) 69 pixels, (f) 99 pixels. 


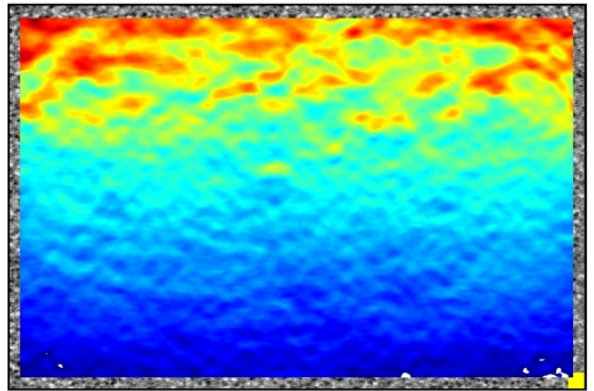

(a)

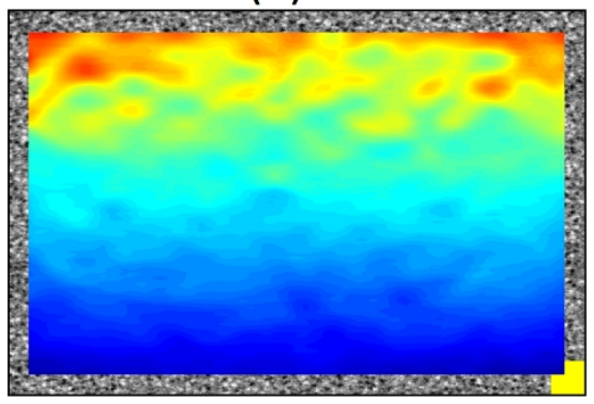

(b)

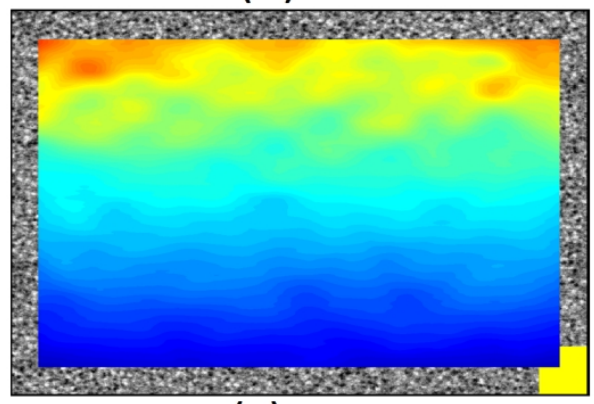

(c)

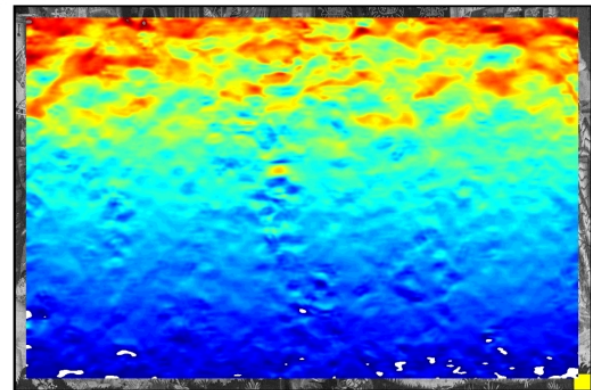

(d)
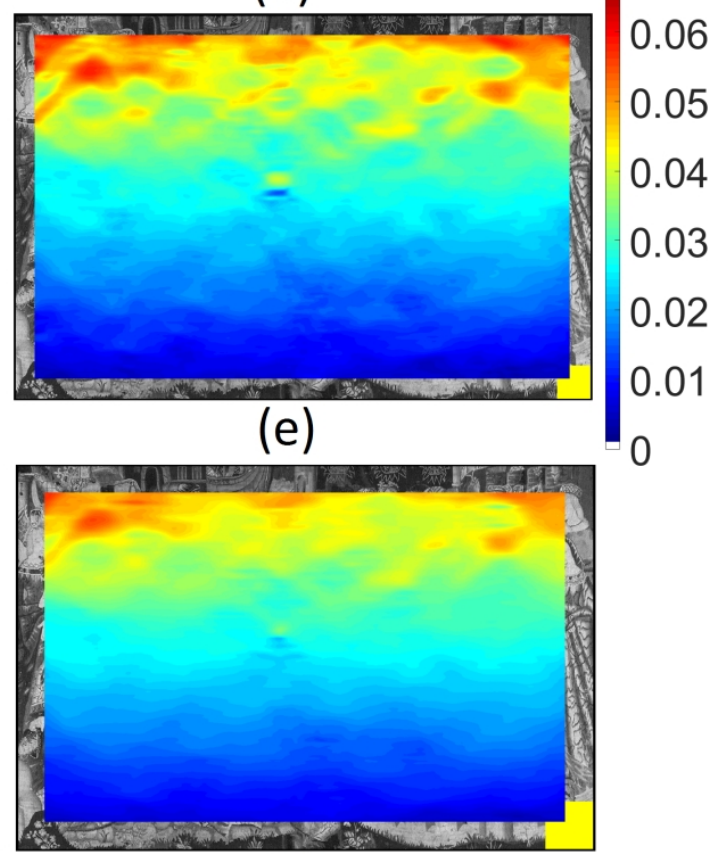

(d)

Figure 13: Right: The DIC-measured LE22 strains deformed using predicted simulation strains from heterogeneous material assignment (Case 2): (a-c) using speckle-pattern images to track deformation and subset sizes shown by yellow corner square, of (a) 33 pixels, (b) 69 pixels, (c) 99 pixels. (d-f) using tapestry image to track deformation and subset sizes of (d) 33 pixels, (e) 69 pixels, (f) 99 pixels 


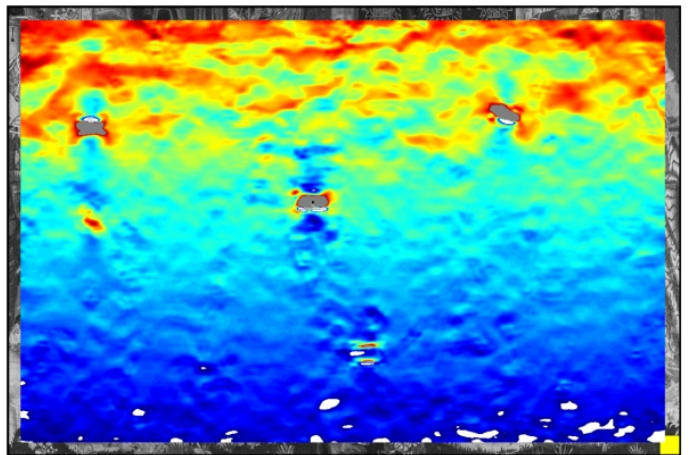

(a)

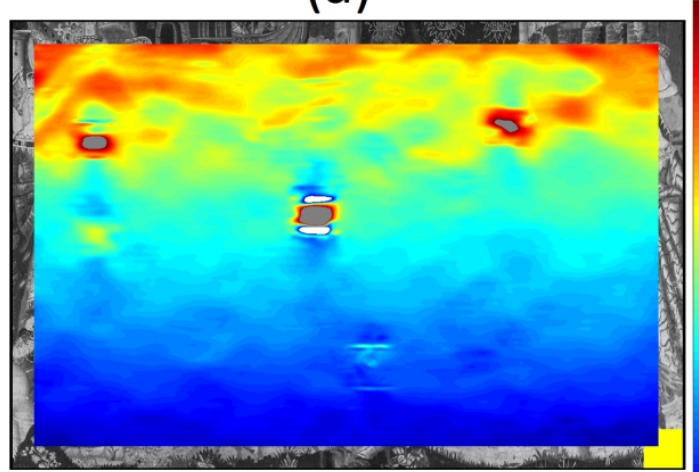

0.07

0.06

0.05

0.04

0.03

0.02

(b)

0.01

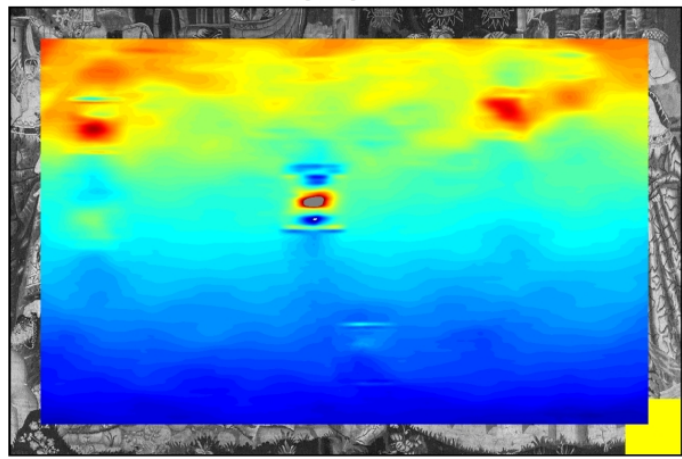

(c)

Figure 14: The DIC-measured LE22 strain fields for the images deformed using the computer model with the heterogeneous material assignment and five tears for the tapestry-pattern images by the applied subset sizes of (a) 33 pixels, (b) 69 pixels, (c) 99 pixels. 


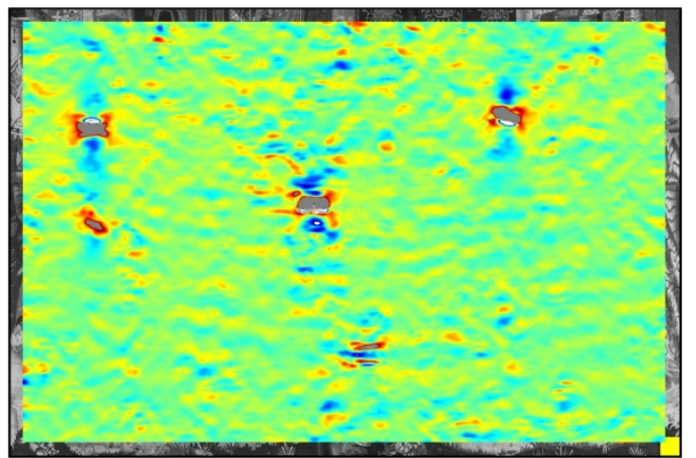

(a)

0.03

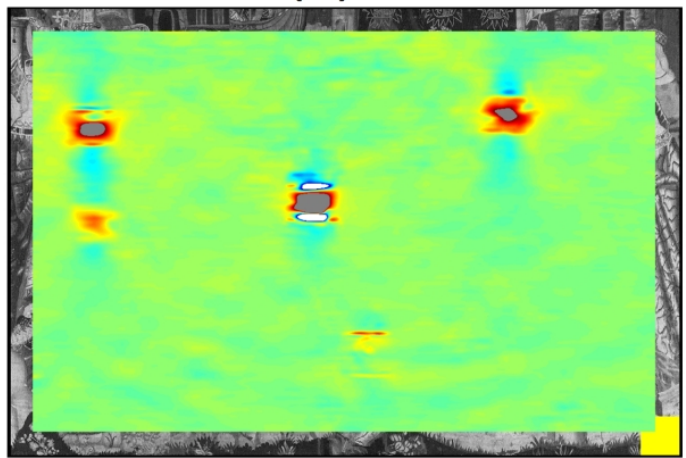

0.015

0

$-0.015$

(b)

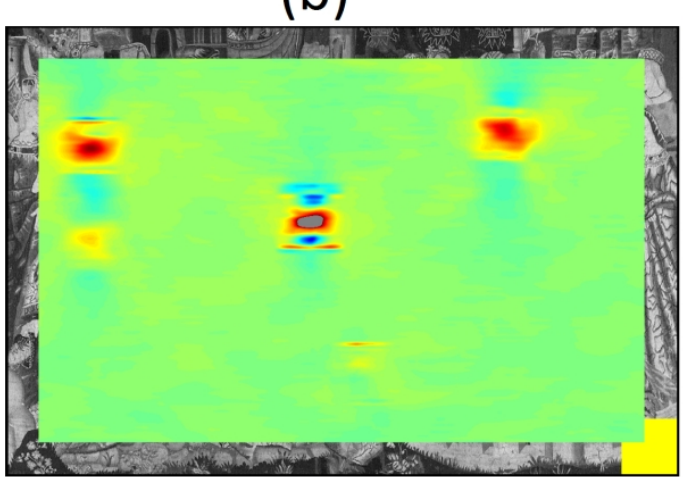

(c)

Figure 15: The difference between the DIC-measured LE22 strain fields for the images deformed using the computer model with the heterogeneous material assignment and five tears and the Figure 13 for the tapestry-pattern images by the applied subset sizes of (a) 33 pixels, (b) 69 pixels, (c) 99 pixels. 

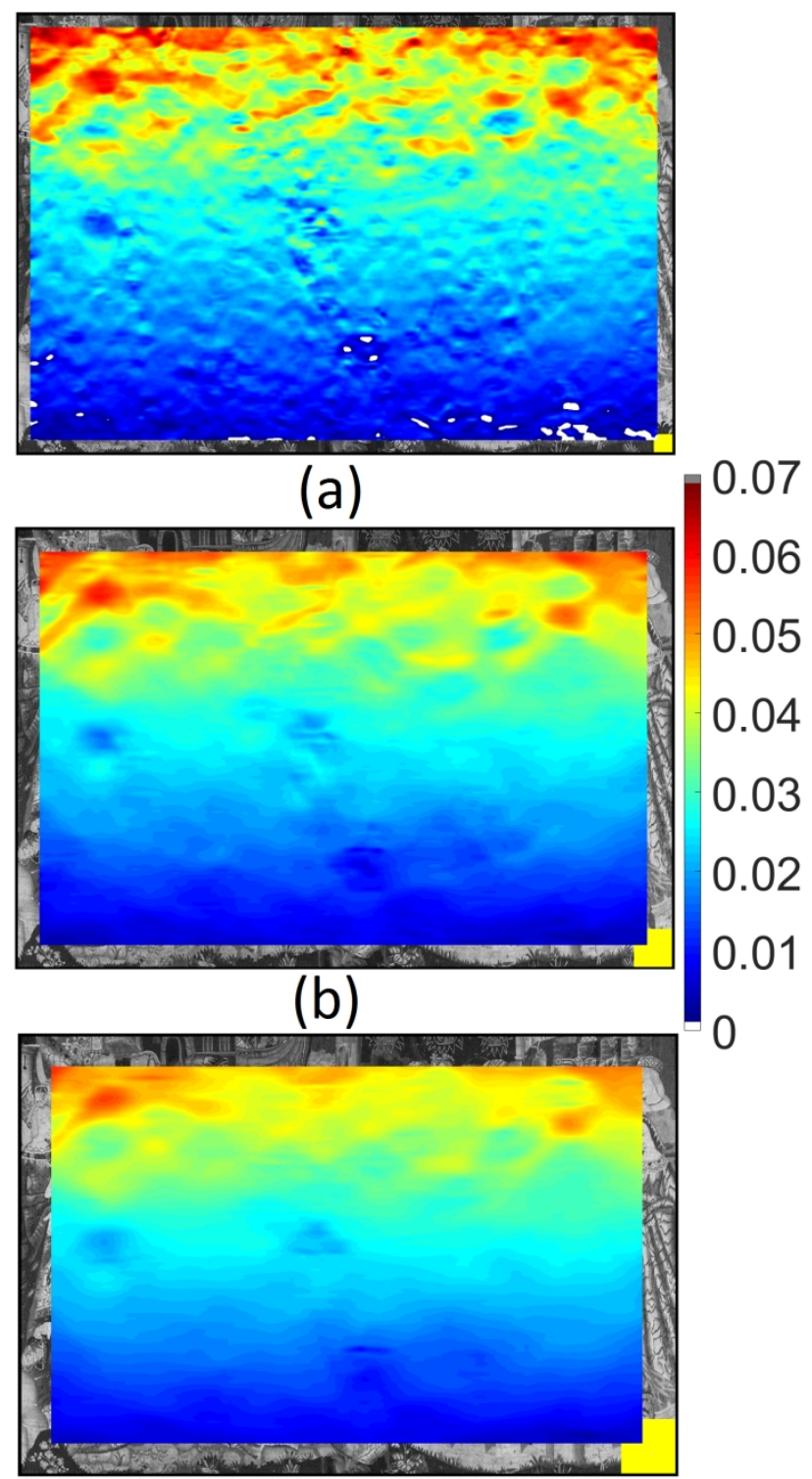

(c)

Figure 16: The DIC-measured LE22 strain fields for the images deformed using the computer model with the heterogeneous material assignment and five patch support treatments for the tapestry-pattern images by the applied subset sizes of (a) 33 pixels, (b) 69 pixels, (c) 99 pixels. 

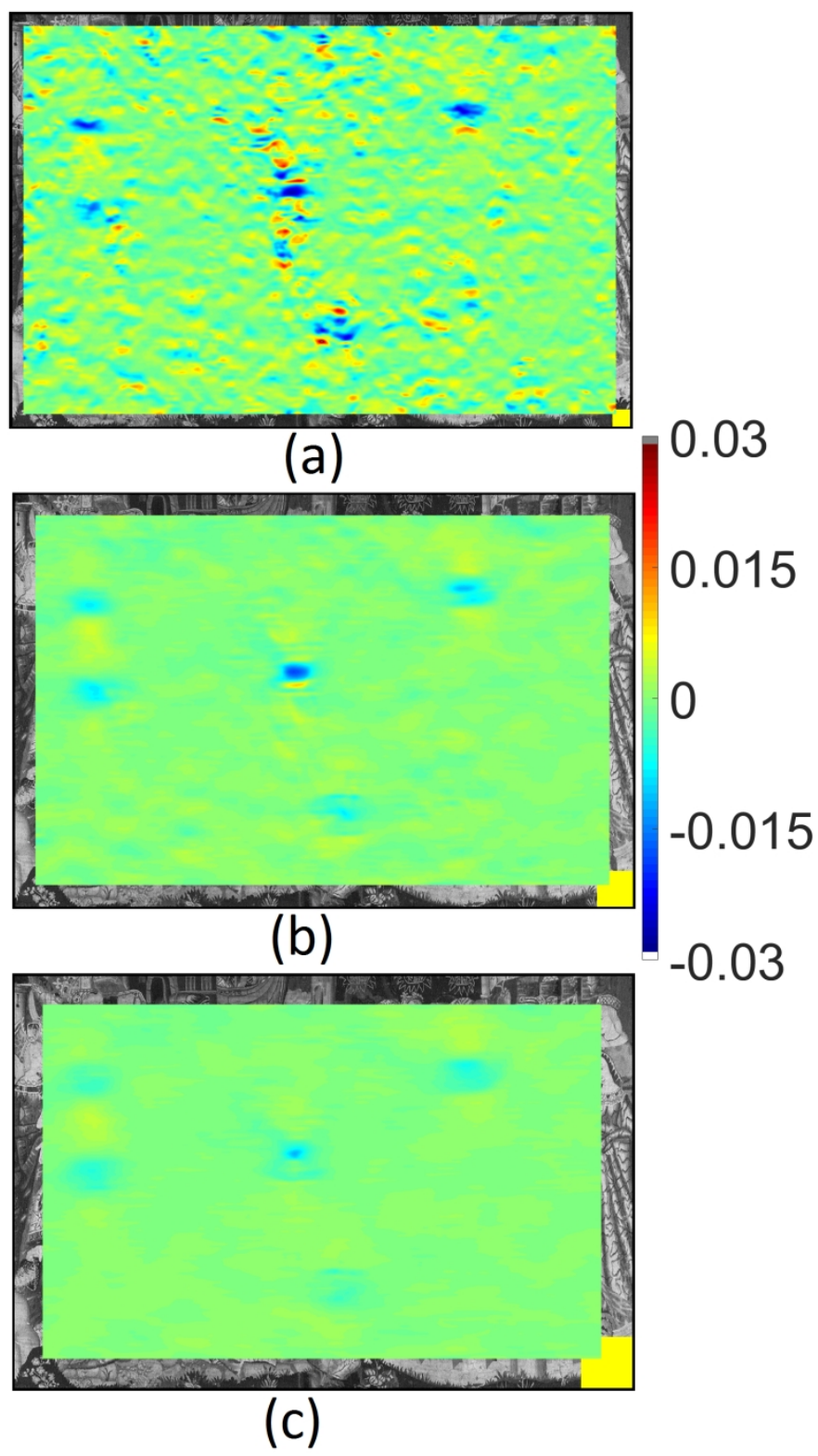

Figure 17: The difference between the DIC-measured LE22 strain fields for the images deformed using the computer model with the heterogeneous material assignment and five patch support treatments and Figure 12 for the tapestry-pattern images by the applied subset sizes of (a) 33 pixels, (b) 69 pixels, (c) 99 pixels. 


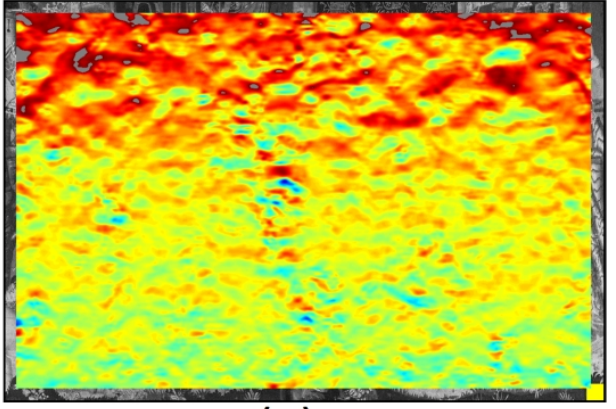

(a)

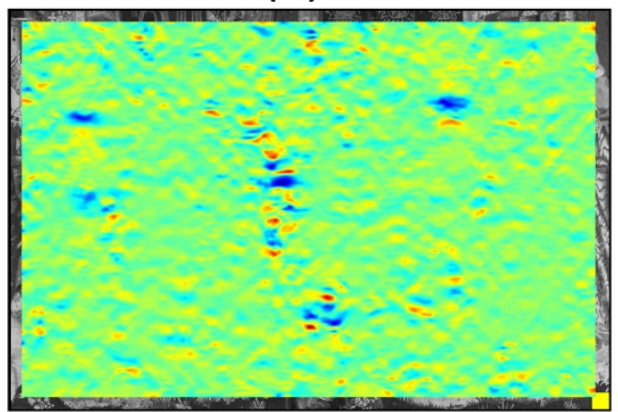

(c)

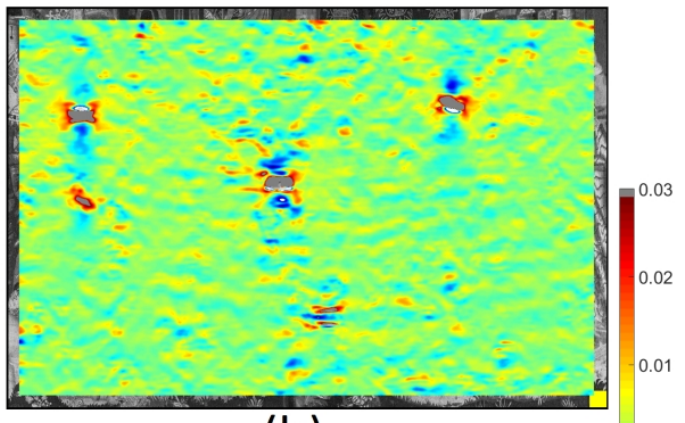

(b)

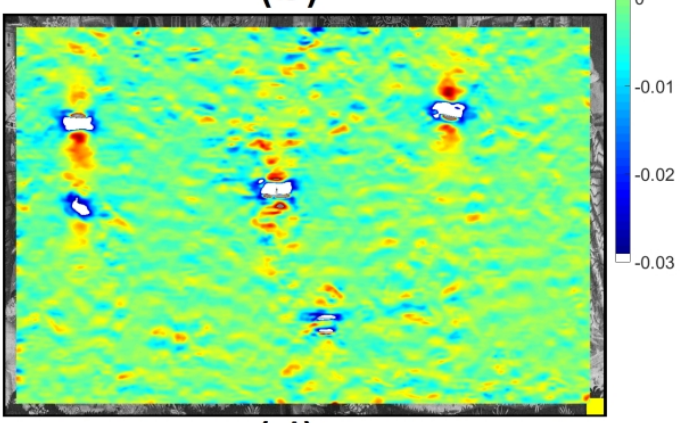

(d)

Figure 18: DIC difference plots for the images with the tapestry-patterns and applied subset size of 33 pixels: (a) Homogeneous strain field subtracted from heterogeneous (join line) strain field, (b) heterogeneous strain field subtracted from tear strain field, (c) heterogeneous strain field subtracted from patched strain field, (d) the tear strain field subtracted from the patched strain field. 


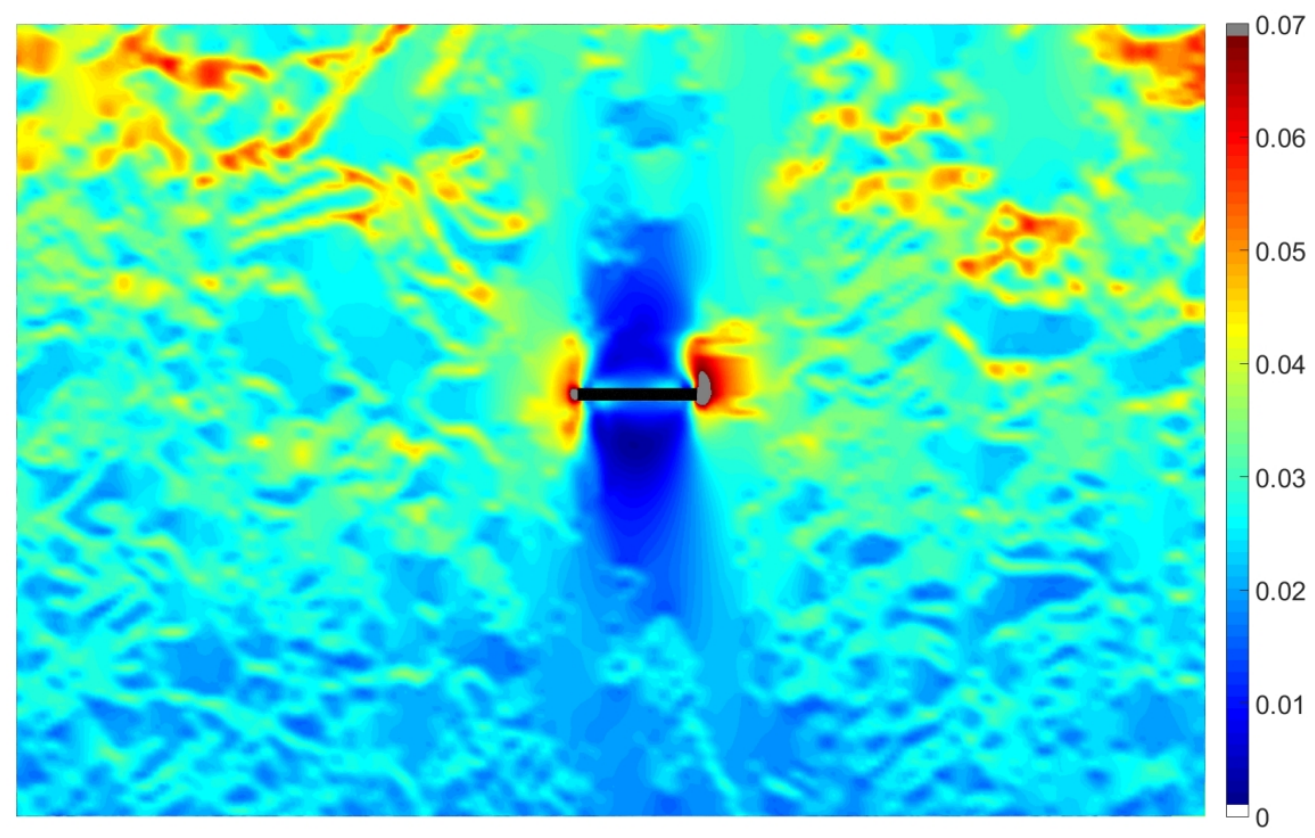

Figure 19: The Abaqus LE22 strain contours for the selected region of interest 


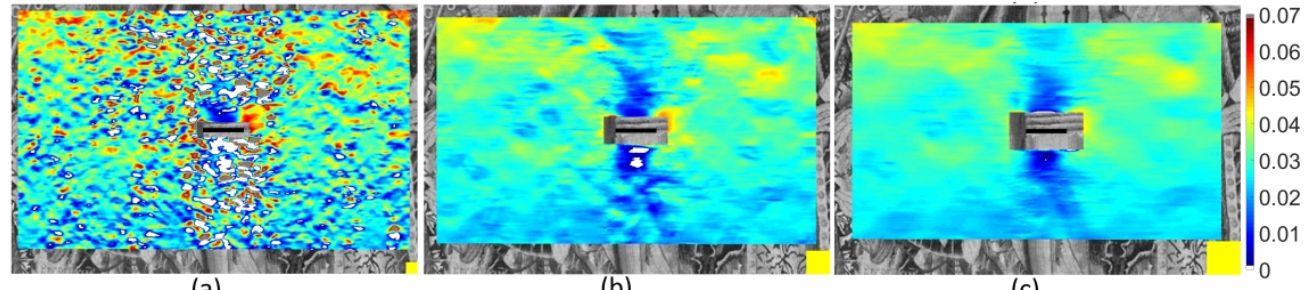

(a)

(b)

(c)

Figure 20: The DIC-measured LE22 strain fields for the magnified deformed images using the tapestrypattern images by the applied subset sizes of (a) 33 pixels, (b) 69 pixels, (c) 99 pixels. 


\title{
Evaluating the Use of Digital Image Correlation for Strain Measurement in Historic Tapestries using Representative Deformation Fields
}

\author{
Alsayednoor, J. ${ }^{a}$, Harrison, P. ${ }^{a,}{ }^{*}$, Dobbie, M. ${ }^{c}$, Costantini, R. ${ }^{b}$ and Lennard, F. ${ }^{b}$ \\ a School of engineering, University of Glasgow \\ ${ }^{b}$ School of Culture and Creative Arts, University of Glasgow \\ 'Glasgow Museums \\ ${ }^{*}$ Corresponding Author (Philip.harrison@glasgow.ac.uk)
}

\begin{abstract}
Keywords: tapestry, digital image correlation, finite element modelling, full-field monitoring,
\end{abstract} textile conservation

\section{Abstract}

An analysis technique to assess the viability of digital image correlation (DIC) in tracking the full field strains across the surface of hanging historic tapestries, is presented. Measurement uncertainty related to the use of the inherent tapestry image in tracking displacements is investigated through use of 'synthetic' deformation fields. The latter are generated by mapping the details of a given tapestry image into finite element analyses. The combination of self-weight loading, material non-linearity and image specific heterogeneity (related to slit-stitching, damage and patch-restorations), serve to generate a bespoke deformation field complex enough to assess the reliability of DIC measurements. Accuracy is evaluated by comparing measured results with the original known deformations. The technique demonstrates that the optimum imaging settings and the choice of subset size for DIC analysis are strongly influenced by the tapestry 
image and the goal of the measurement, they are found using a compromise between conflicting objectives: minimising measurement error while maximising resolution.

\section{Introduction}

Historic tapestries are an important part of a nation's cultural heritage. Over time, exposure to mechanical stresses and strains can lead to damage and the subsequent need for expensive repair and restoration. In the UK alone over $f 3 \mathrm{~m}$ is allocated annually to the conservation of historic tapestries [Lithgow, 2013]. In the attempt to both conserve and display such tapestries, conservators and curators must make choices that influence both the load and load distributions acting across these fragile works of art.

The effects of loading on a hand-woven historic tapestry are difficult to anticipate due to its inherently heterogeneous mechanical properties, resulting from its complex mesoscale structure. Tapestries consist of undyed warp threads and coloured weft threads (which due to their close packing, completely cover the warps). The weaver typically works on one weft colour at a time, resulting in discontinuities of the weft yarns (see Figure 1). Frequently, a gap or 'slit' is left during weaving and subsequently stitched together; a technique known as 'slit stitching', this creates a relatively weak join. Over time, it is very common for slit stitching to fail (and later to be repaired). Slit stitching is found across both large and small slits all over a tapestry and commonly runs horizontally when the tapestry is hung, creating likely regions for subsequent damage and tears. 


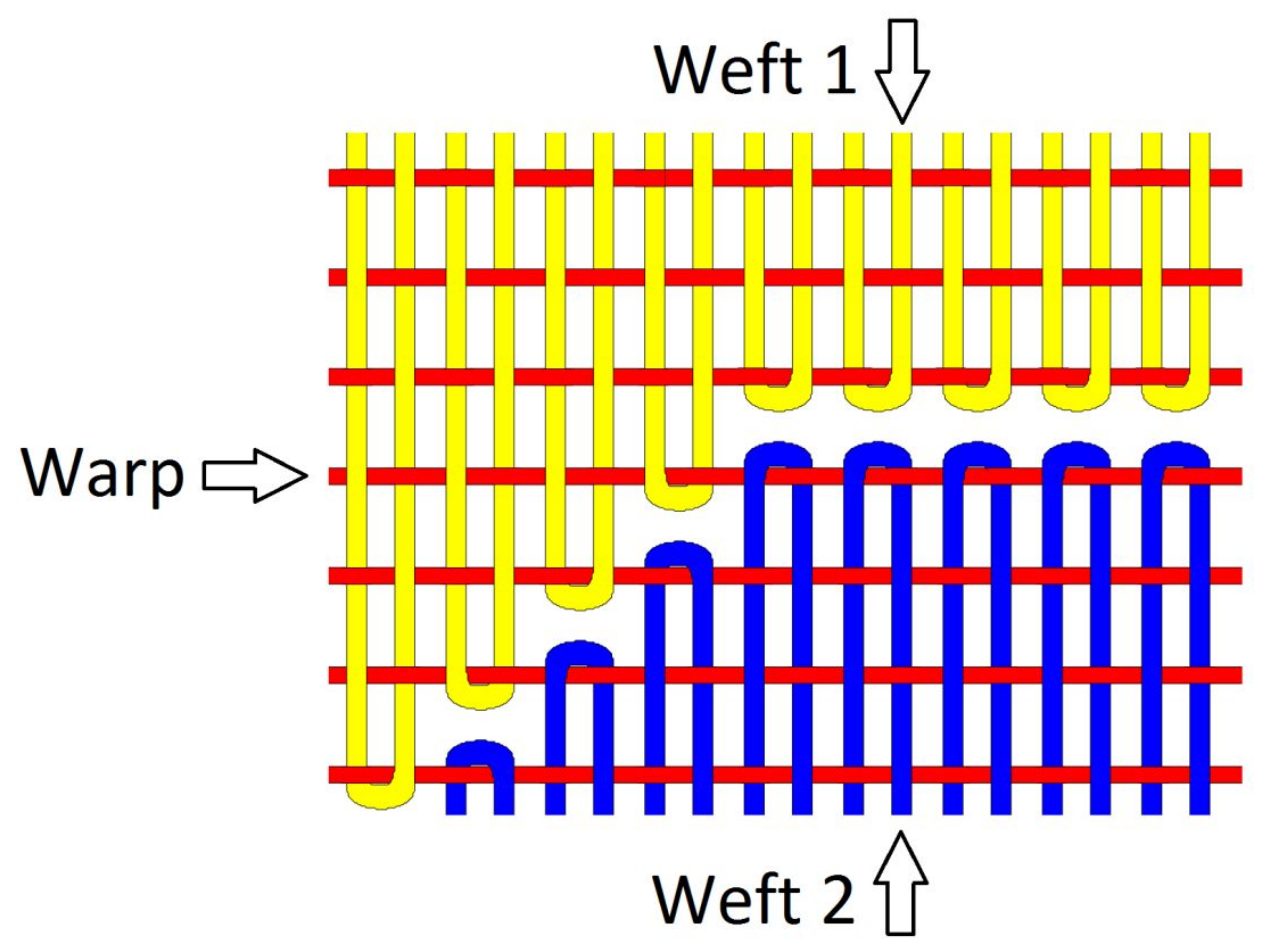

Figure 1. Schematic of the slits occurring between different coloured weft regions of the tapestry

In addition to the mechanical complexity attributable to joining methods, conservation techniques such as mechanical reinforcement of tears and holes via stitching, reweaving or the application of backing patches [Lennard and Hayward, 2006], as well as the choice of display method such as vertical hanging from multiple fixed points, attachment along a continuous strip or support using sloping boards [Lennard and Hayward, 2006] all inevitably influence the stress and stress distributions acting on a tapestry in complex and possibly undesirable ways. Use of different stitching threads (e.g. polyester, wool or cotton) and the stitching pattern (e.g. size, density, style) can all significantly influence the mechanical behaviour and longevity of a repair [Benson et al. 2014] and interventions in one part of the tapestry can lead to unintended increased stresses in neighbouring regions. Typically, the choice of mechanical conservation and display technique is based on practical shared experience with little reliance on full-field strain measurement or without the insights offered by computational modelling. The goal of this work is to help determine how the application of state-of-the-art engineering technology can inform conservation and display strategies. In developing a more robust understanding of the consequences and efficacy of the wide range of display and conservation intervention strategies, 
we aim to inform and improve existing practice and ultimately reduce the damage and resulting high costs involved in conserving historic tapestries.

\subsection{Use of DIC in conservation}

Digital image correlation (DIC) is a convenient non-contact full-field optical technique [Sutton et al. 2009] that has already been trialled as a method to measure strains acting across historic tapestries [Lennard and Dulieu-Barton, 2014]. The aim is to investigate the influence of different display methods and conservation interventions on the resulting local and global strain distributions across the tapestry. For optimum performance, DIC requires a detailed, isotropic and non-periodic image, such as a random speckle pattern, printed or sprayed onto the surface of an object to produce accurate full-field displacement data [Sutton et al. 2009]. In most engineering applications, the speckle pattern is typically applied to the target object using paint [e.g. Harrison et al. 2017], though clearly this is not an option when analysing valuable historic tapestries. Consequently, previous attempts to use DIC with tapestries [Khennouf et al. 2010] (and also oil paintings [Dureisseix et al. 2011, Gauvin et al. 2014]) have used the inherent image and texture of the artwork itself to track displacements. Nevertheless, while promising, such an approach can lead to significant hidden errors in the DIC measurements, potentially large enough to throw doubt on interpretations of the results.

\subsection{Understanding the experimental error due to use of the inherent tapestry image in tracking the strains}

When performing any experimental measurement, it is important to understand the associated error and confidence in the resulting data. DIC algorithms can often provide a full field strain measurement, even when using a non-ideal target pattern, but the accuracy of the measurement in such cases is far from guaranteed. Quantifying the error in DIC is a non-trivial matter, even when using purpose-designed speckle patterns to track deformations. Factors such as the quality of the imaging system, the signal to noise ratio, the correlation algorithm, the influence of nonplanar surface geometries and out-of-plane motion and of course, the quality and properties of the speckle pattern itself are all important [Amiot et al. 2013, Ke et al. 2011, Schreier et al. 2000, Pan et al. 2008, Sutton et al. 2008]. See Pan, 2018 for a comprehensive recent review on this matter. 
The complexity of the strain field and the size of the region of interest are important considerations when choosing the optimum DIC setup. A localised region around a specific tear or repair will require a different setup compared with a more extensive region of interest, covering much of the tapestry and potentially possessing a more undulating, out-of-plane surface morphology. In this paper, a technique to highlight the likely limitations or the potential utility of a given tapestry is demonstrated, and employed to estimate the optimum DIC set-up parameters prior to conducting experiments. This is the first time that the accuracy of using DIC to directly monitor the full field strains across historic tapestries has been systematically analysed. Previous attempts to use DIC with historic tapestries (e.g. Khennouf et al. 2010) while ground-breaking, paid little attention to the errors that naturally arise when using the inherent tapestry image to track displacements and strains, leading to unknown and potentially significant errors in the results.

Finally we note that error due to non-planarity of the target object is sometimes also an important factor in generating errors but is not considered in this investigation. For example, if there is significant non-planarity of the tapestry due to out-of-plane undulations [Lou et al. 1993, Orteu, 2009, Tang et al. 2010] or if the tapestry moves due to circulating air currents during the experiments further error can creep into the DIC measurements. Rather than introducing a detailed consideration of this particular influence on the optimum experimental DIC setup this paper deals only with flat specimens. Future experimental work following on from this investigation will aim to constrain any target tapestry to 2-D, avoiding the not insignificant practical issues associated with long-term usage of stereoscopic 3-D systems in public spaces.

\section{Method}

\subsection{Error assessment}

A common approach to error assessment across planar surfaces is to perform a numerical transformation of a reference image (i.e. to stretch and rotate the reference image) by a known amount and to then compare this known deformation with that measured by the DIC system [Bornert et al. 2012]. To investigate DIC measurement errors for heterogeneous deformations, Lecompte et al. 2006, Lava et al. 2009, 2010 and Wang et al. 2012, all used the technique of interpolation-based image transformation using displacement fields predicted from finite 
element simulations. These studies revealed a strong relationship between the subset-size, the quality of the speckle pattern and the accuracy of the measured displacement fields. In this investigation we aim to automatically and quickly generate a bespoke full-field synthetic strain field for any given tapestry that bears some probable relation to reality including likely sites for concentrated strains and damage. The simulations are by no means designed to be accurate and truthful mechanical predictions. Such an endeavour would be an extremely complex and timeconsuming task involving detailed material characterisation and dedicated constitutive modelling. Nevertheless, this difference between the 'true' and the 'representative' strain field is not an issue for our purposes; namely, to generate an informed synthetic strain field and use this to provide an indication of the viability of using DIC to monitor the strains for a given tapestry. The approach employed in this investigation is summarised in Figure 2.

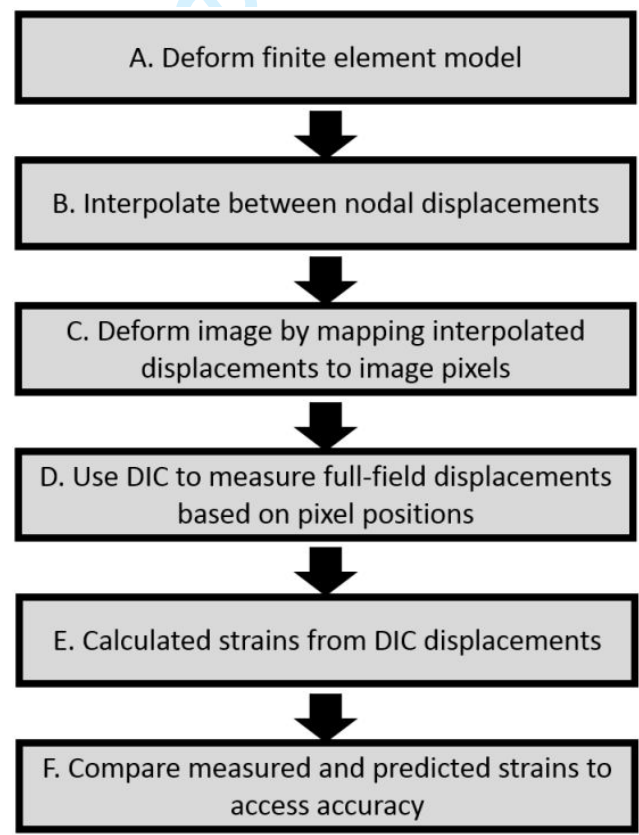

Figure 2. Flow chart showing DIC assessment process.

To illustrate the method and demonstrate the image-dependent effectiveness of DIC in analysing historic tapestries, an example tapestry image is considered (see Figure 3). This specific tapestry was chosen due to its complex and intricate image, a favourable property when aiming to conduct DIC. In order to evaluate the potential of image-based DIC in measuring full-field strains, 
a representative tapestry model is subject to progressively more complex, heterogeneous strain fields. The motivation behind each additional layer of complexity is related to the:

1. Hanging boundary conditions used to display the tapestry (different hanging techniques can alter the self-weight induced stress distribution across the tapestry across the tapestry)

2. Structure-induced heterogeneity due to weakness along stitched slits

3. Damage (e.g. tears and holes due to failed stitching - see Figure 4)

4. Conservation treatment (e.g. patching)

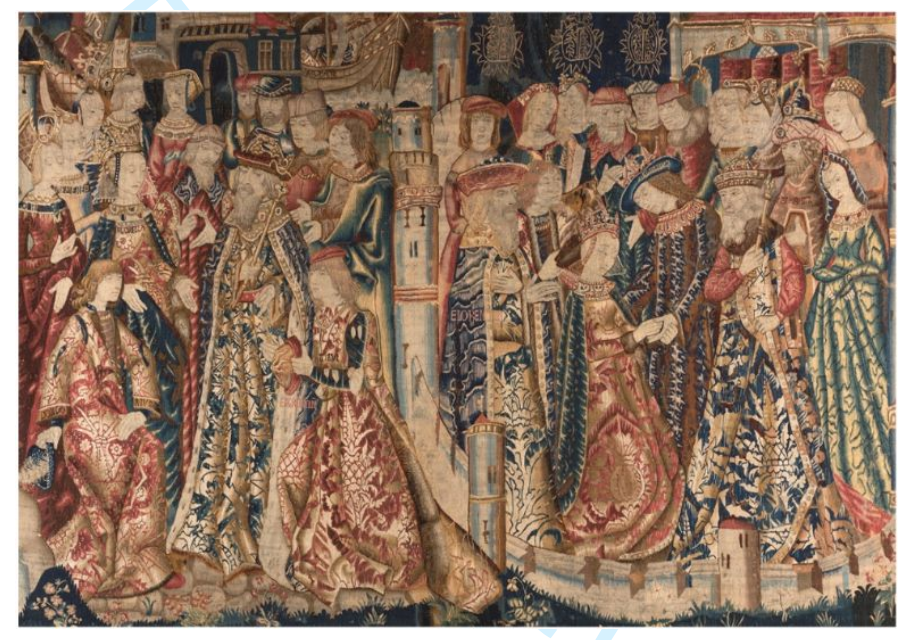

Figure 3. Two Episodes from a Chivalric Romance, Perhaps the Tale of Florence of Rome, c. 1480, 2.10 × 3.0m, The Burrell Collection, Glasgow Museums, (C) CSG CIC Glasgow Museums Collection.

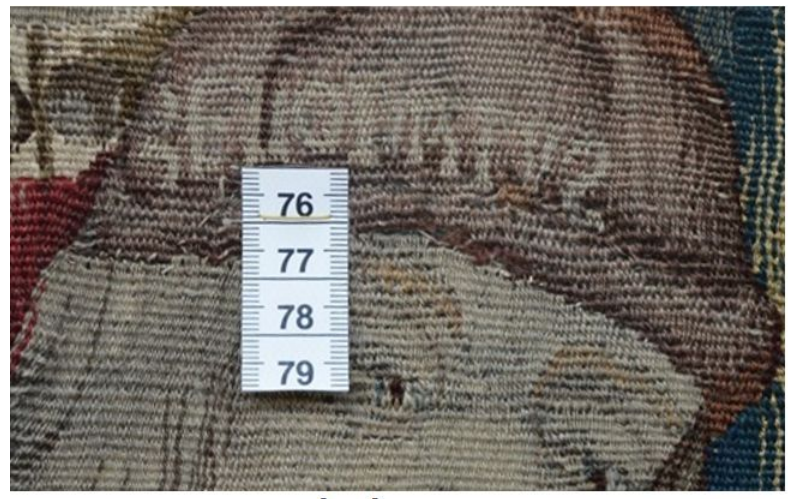

(a)

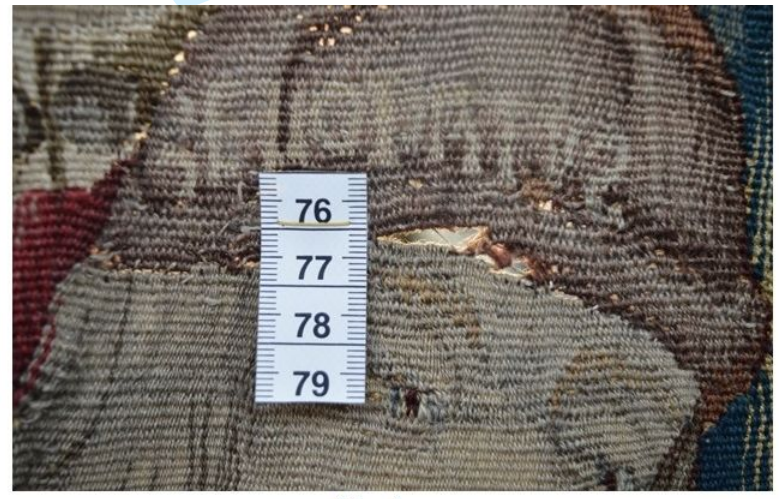

(b)

Figure 4: A close up from Figure 3; the effect of self-weight loading on damage propagation (a) just before and (b) two weeks after unsupported tapestry display. Velcro fastener was used to hang the tapestry 
For reference, the most accurate DIC parameters were selected in the software, including an optimised 8-tap interpolation shape function, with normalised squared differences and Gaussian weights, together with exhaustive search, low pass filter and incremental correlation. A subset step size of 1 was used and the software's default options were applied for thresholding. These parameters are set to produce the greatest accuracy and were kept constant throughout the investigation to focus on the effect of changing subset size. The latter is perhaps the most difficult parameter to choose when conducting DIC as it involves a trade-off between measurement accuracy and resolution of detail in the strain field (as will be demonstrated later in this investigation). The strain fields are analysed first using an ideal speckle pattern and next using the inherent tapestry image; both techniques employ a range of subset sizes to determine the optimum subset size for a given strain measurement. The reason for using both an ideal speckle pattern and the inherent tapestry image is to differentiate between the errors introduced by the node to pixel displacement mapping (Steps B \& C in Figure 2). Because the number of nodes in the finite element mesh is different (less) than the number of pixels in the tapestry image, interpolation is required to map the displacement field from the simulation to the pixels in the tapestry image. Cubic interpolation is used in this study, introducing a small but unavoidable systematic error [Schreier et al. 2000, Bornert et al. 2012]. To mitigate this interpolation bias particularly for noisy data, pre-smoothing process using a Gaussian low-pass filter is recommended, as proposed in Pan, 2013. To decouple this systematic interpolation error from the tapestry's image-related DIC error, a digitally-generated random speckle pattern is created using the Perlin noise technique [Orteu et al. 2006] (see Figure 5). 


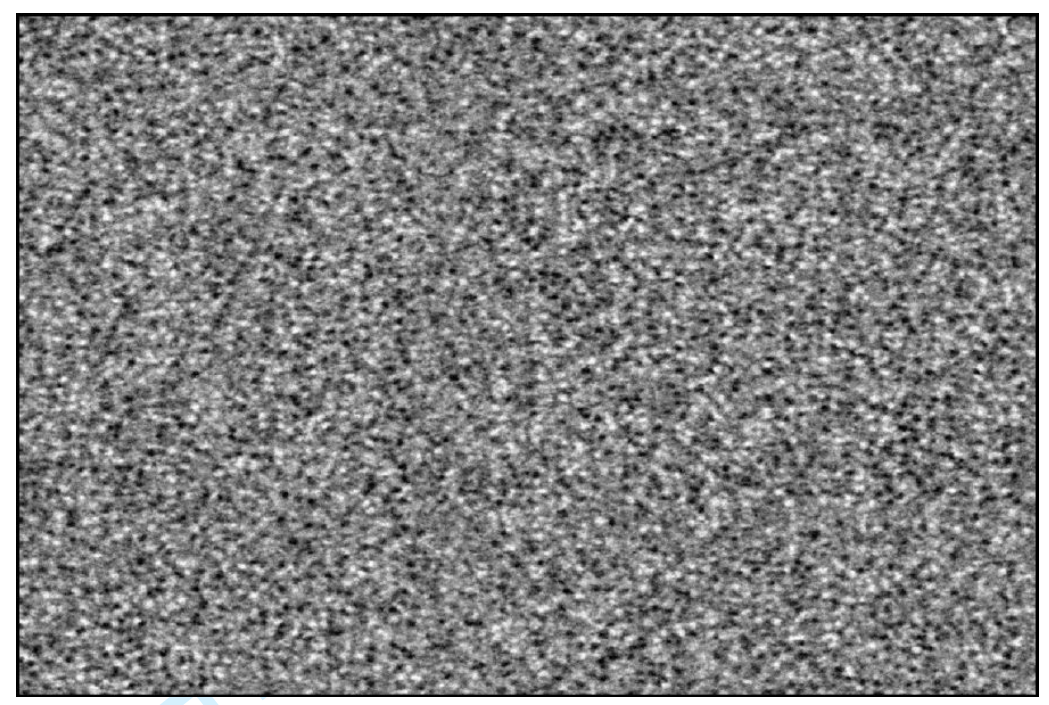

Figure 5: Digitally generated using Perlin speckle pattern with identical dimensions to the corresponding tapestry The speckle-pattern and tapestry images are both deformed using the same interpolated heterogeneous displacement field. Consequently, both the speckle pattern and the tapestry images contain the same error due to interpolation. The difference in accuracy between the results produced by analysis of the speckle and tapestry images is therefore entirely attributable to the use of the tapestry image in tracking the deformation and is highlighted in Section 3.1 by directly comparing results from both types of target tracking image. As discussed, the main focus of the investigation is in demonstrating a technique to evaluate the suitability of a given inherent tapestry image in measuring the full-field strains occurring across a tapestry, due to, for example, the tapestry's inherent mechanical heterogeneity, hanging conditions, tapestry damage and conservation treatments.

\subsection{Material properties}

Finite element simulations are used to provide an estimate of strains that might be representative of those occurring in an actual tapestry. The true mechanical behaviour of historic tapestries is extremely complex. Aside from the usual anisotropic response of fabrics, in which each property is almost entirely independent from all other properties (e.g. tensile, shear, torsion and flexural properties in the warp and weft directions are all uncoupled) [Hu 2004, Harrison 2017], other factors such as aging and degradation, humidity and creep all influence material properties [Khennouf et al. 2010] making accurate modelling of tapestries a significant challenge. To avoid this complexity, a pragmatic approach is followed that relies on the fact that tapestries 
are predominantly loaded in one direction during display, i.e. in the direction of gravity due to their own self-weight. For this limited case, an isotropic mechanical model can provide a reasonable first order estimate of the strains likely to occur within any given homogeneous region of a tapestry assuming its density and dimensions are known. Experimental data on the representative uniaxial properties along the highly crimped weft direction of the fabric are used as almost all tapestries are hung such that the weft yarns support the tapestry [Khennouf et al. 2010]. To this end a series of mechanical tests were conducted and are reported in Section 2.2.1.

\subsubsection{Uniaxial tensile properties along the weft yarns}

Twenty rectangular test specimens measuring $10 \times 40 \mathrm{~mm}$, taken from seven different historic tapestries were used (each represented by its own colour in Figure 6). The thickness of the specimens was approximately $1 \mathrm{~mm}$ and so a thickness of $1 \mathrm{~mm}$ was used in calculating the nominal tensile stress (ignoring subsequent changes in thickness resulting from the tensile loading). All uniaxial tensile tests were performed along the weft direction. The tests were carried out using an Instron 5544 test machine, fitted with a $1 \mathrm{kN}$ loadcell, at an extension rate of 10 $\mathrm{mm} / \mathrm{min}$. The nominal stress-strain data and densities are shown in Figure 6 . The stress-strain curves are clearly highly non-linear. For this reason, and because for the purpose of this investigation a one-way loading is sufficient, a hyper-elastic material model was used to represent the data.

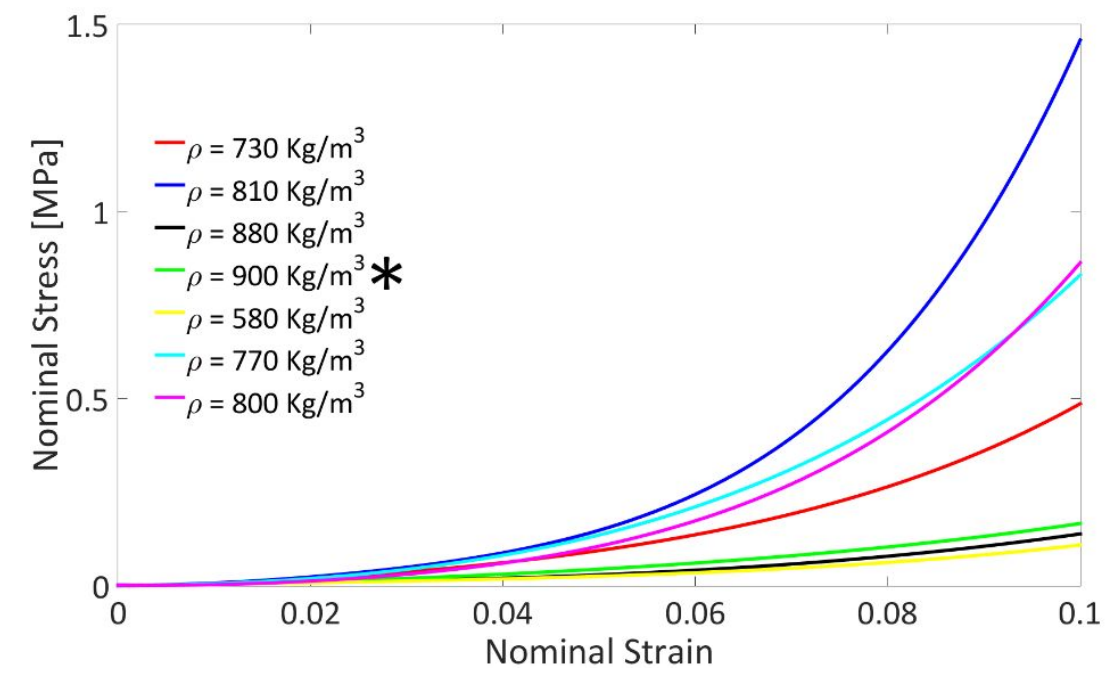

Figure 6: Nominal stress-strain curves for 20 specimens extracted from 7 historic tapestries (different colour code represents different historic tapestry). The selected curve is highlighted by the star symbol. 
A stress-strain curve with low stiffness and high density was used as input for the simulations (again favourable choices in generating larger, more measurable strains). The Abaqus material evaluation tool [Abaqus v6.14, User Manual] was used to fit the parameters of an isotropic 'Ogden N2' model [Abaqus v6.14, User Manual, Section 22.5.1], giving the following parameters

$U=\sum_{i=1}^{N} \frac{2 \mu_{i}}{\alpha_{i}^{2}}\left(\bar{\lambda}_{1}^{\alpha i}+\bar{\lambda}_{2}^{\alpha i}+\bar{\lambda}_{3}^{\alpha i}-3\right)+\sum_{i=1}^{N} \frac{1}{D_{i}}\left(J^{e l}-1\right)^{2 i}$

Eq1.

$\mu_{1}=1.68 \mathrm{MPa}, \mu_{2}=-1.62 \mathrm{MPa}, \alpha_{1}=9.35, \alpha_{2}=7.32, D_{1}=0, D_{2}=0$

In the above equation, $\bar{\lambda}_{i}$ represent the deviatoric principal stretches, $J^{e l}$ is the elastic volume ratio, $N$ is the material parameter; where $\mu_{i}, \alpha_{i}$ and $D_{i}$ denote temperature-dependent material parameters. Only uniaxial data were used in the fitting process, this was considered enough to provide reasonable strains with which to evaluate the DIC measurements.

\subsection{Generating an image-dependent heterogeneous strain field}

A known heterogeneous strain field is a useful tool to determine the optimum DIC subset size [Lava et al. 2010]. On the one hand, use of a relatively large subset size means that each subset contains more information. Larger subsets are therefore more likely to be unique and identifiable, making them easier to track following deformations. However, implicit in the DIC technique is the assumption that the deformation within each subset is homogeneous. If the heterogeneity of the applied strain field occurs at length scales smaller than the subset dimensions, then the DIC algorithm is less able to identify the details of the local strain field. When the tracking pattern is not ideal, as in the case of a tapestry image, the problem is exacerbated. Here, larger subsets are preferred to ensure that each subset is unique and identifiable.

In this study, a similar approach to that used by Lecompte et al. 2006 is adopted to artificially deform the reference images of the tapestries using a heterogeneous strain field predicted via finite element simulations. By progressively introducing deformations that represent the distinct origins of the heterogeneous strain field, the ability of DIC to measure these strains using the inherent tapestry image are assessed. A finite element model with the same dimensions as the 
tapestry image was generated within Abqus Explicit ${ }^{\mathrm{TM}}$ and meshed with equal-sized rectangular membrane elements (M3D4R). Isotropic nonlinear hyperelastic material properties, described in Section 2.2.1, were initially assigned to all elements. To stretch the fabric model using its own weight, the Abaqus gravity loading option was utilised.

Curators and conservators can choose a variety of options to display a tapestry [Lennard and Hayward, 2006]. In this investigation, Velcro ${ }^{\circledR}$ fastening is assumed in which the tapestry is secured to a wooden batten (a wooden strip attached to the wall) by stitching on Velcro ${ }^{\circledR} \operatorname{tape.}$ This boundary condition creates vertical loading due to self-weight together with a resulting inward contraction of the upper side-edges of the tapestry due to the Poisson's ratio effect. To simulate this condition, the upper side nodes of the finite element model are constrained along a straight line using an 'encastre' (fully fixed) boundary condition. This boundary condition is used for all simulations in this investigation.

\subsubsection{Modelling slit stitching}

Historic tapestries are woven textiles but boundaries between different coloured weft yarns, known as slits, are joined with stitching (see Section 1). The slit stitching creates a region of relatively low stiffness and is prone to failure [Lennard and Hayward, 2006], see Figure 4. In order to recreate similar heterogeneity in the finite element model of the tapestry, shown in Figure 3, the Matlab Canny edge detection algorithm [Canny, 1986] is applied to detect the boundaries between different coloured regions within the image of the tapestry (see, for example, Figure 7a which is generated from Figure 3). By mapping the results of the edge detection algorithm onto the finite element mesh, elements lying within boundary regions (blue lines in Figure 7a) are flagged as stitched regions and are assigned relatively low material stiffness properties, half that of the native tapestry material measured in Section 2.2.1 $\left(\mu_{1}=0.84 \mathrm{MPa}, \mu_{2}=-0.81 \mathrm{MPa}, \alpha_{1}=9.35\right.$, $\alpha_{2}=7.32, D_{1}=0, D_{2}=0$ ). Note, in reality a combination of joining techniques would probably be used, though for the sake of simplicity only slit stitching is modelled in this investigation. 
(a)

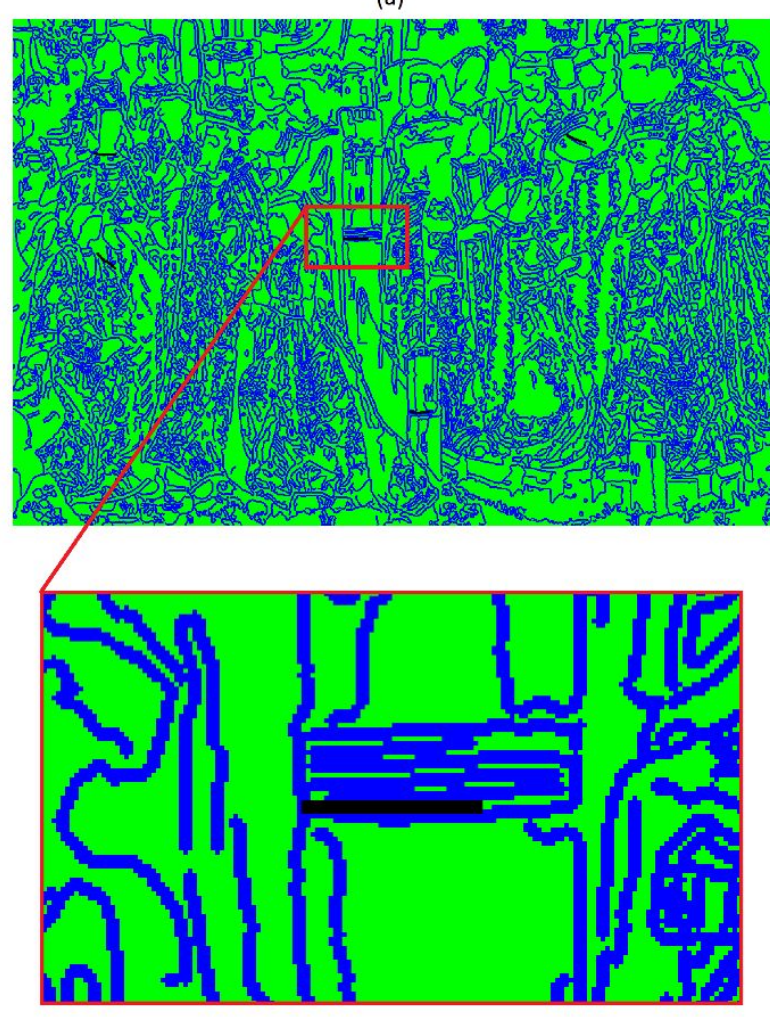

(b)

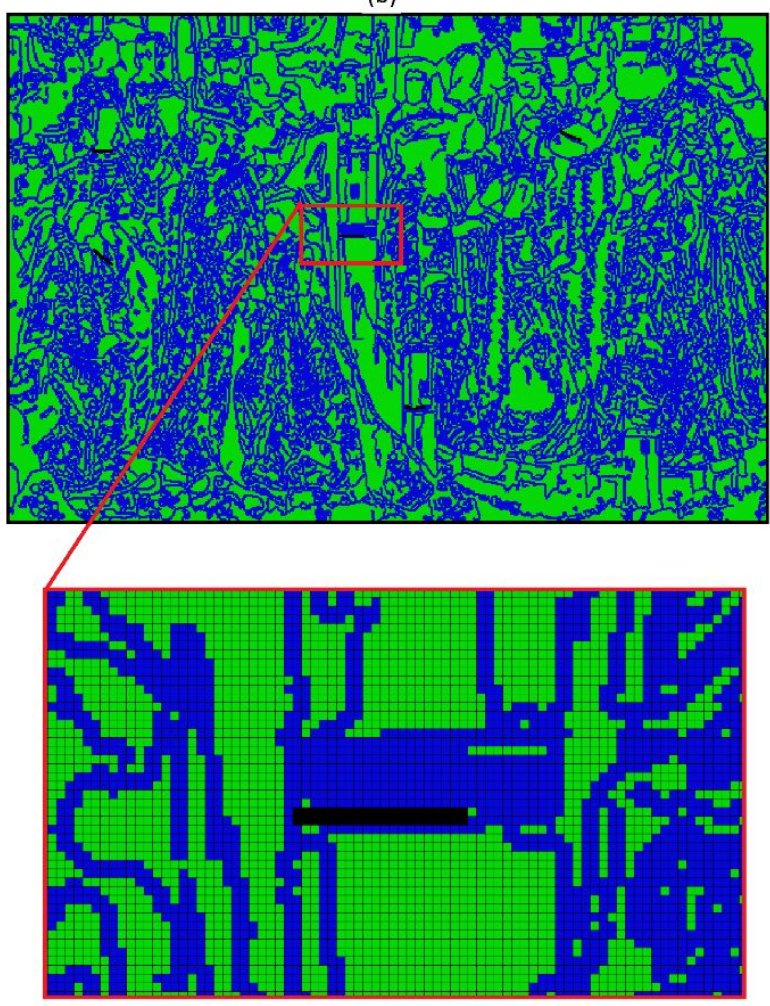

Figure 7: (a) Weak stitched zones, identified using the Canny edge detector (blue colours). Fabric tears were introduced by elimination of the elements along the edges (black colour), (b) the corresponding Abaqus model.

\subsubsection{Modelling tears (Damage along slit stitching)}

Slit stitching in historic tapestries is a likely site for accumulation of micro-damage and can ultimately lead to propagating tears due to the self-weight loading of the fabric. To incorporate representative tears into simulations, five randomly selected sections along existing slit stitching locations were chosen. Tears were modelled simply by deleting the elements along selected sections (see Figure 7).

\subsubsection{Modelling conservation patches}

A common conservation practice for historic tapestry is the application of a full backing or patches of linen or cotton fabric. Stitching through the tapestry into the backing fabric often serves a dual purpose of stabilising the weak and damaged textile structure and contributing to the redefinition of the image. To model patches, rectangular areas large enough to cover the tears were incorporated in the finite element simulations (see Figure 8). Patch elements were 
assigned to relatively stiff material properties, double that of the native tapestry material described in Section 2.2.1 ( $\mu_{1}=3.36 \mathrm{MPa}, \mu_{2}=-3.24 \mathrm{MPa}, \alpha_{1}=9.35, \alpha_{2}=7.32, \mathrm{D}_{1}=0, \mathrm{D}_{2}=0$ ).

(a)

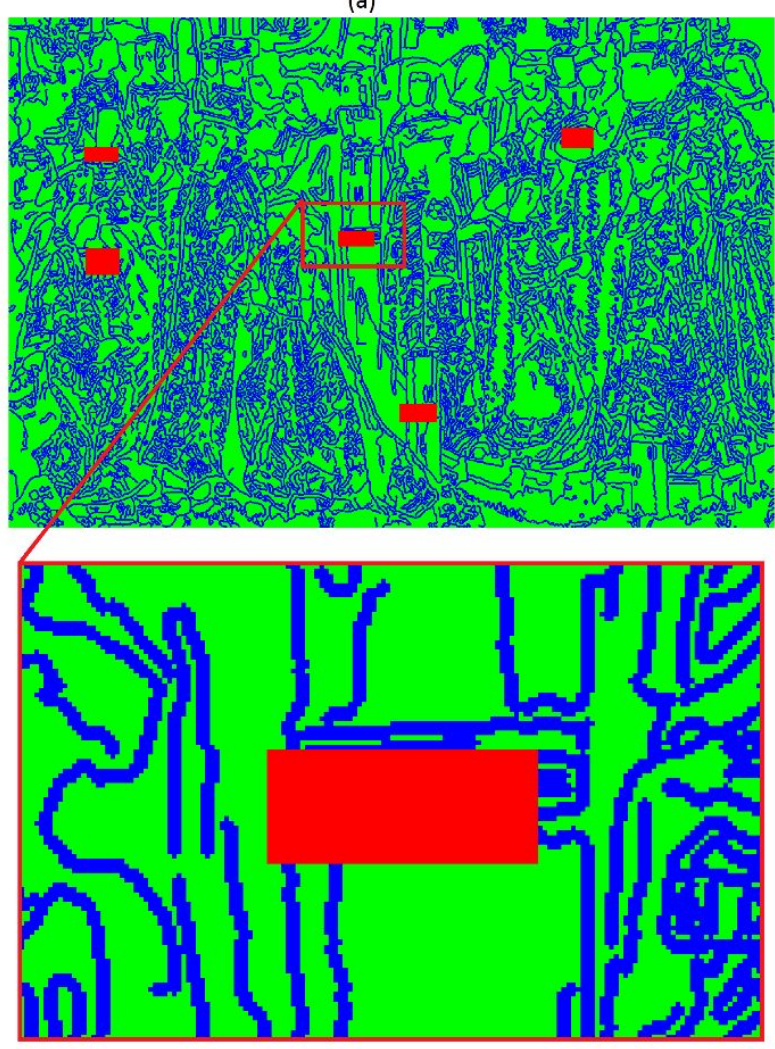

(b)
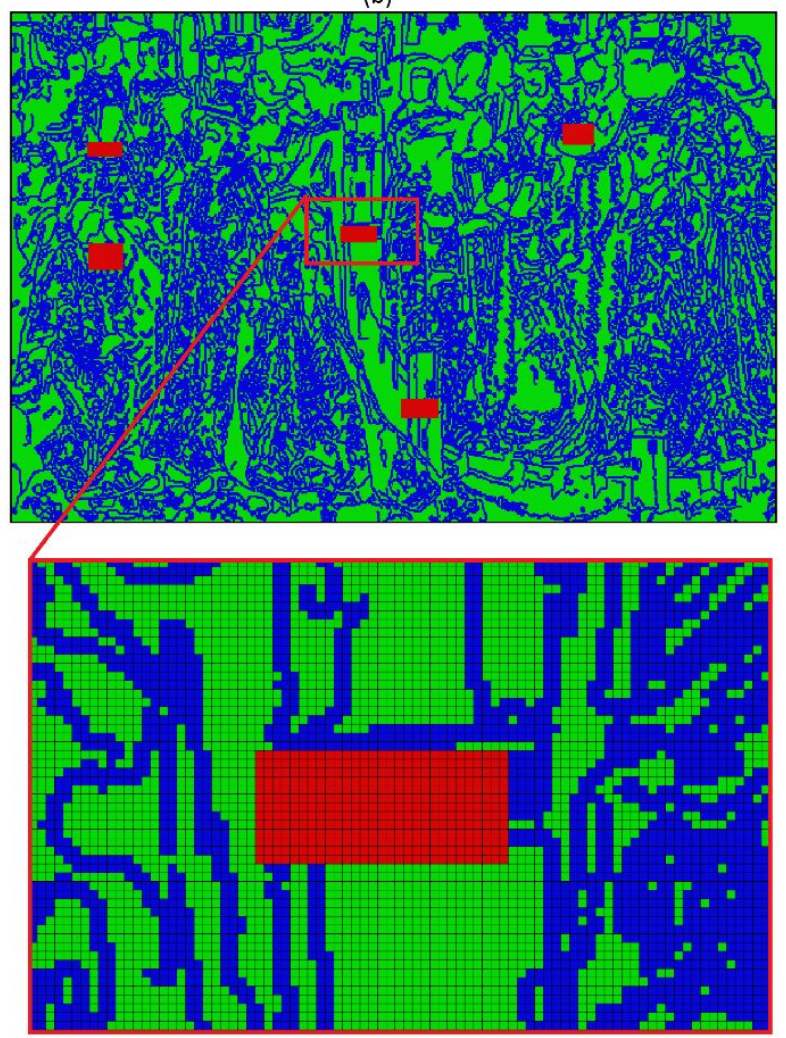

Figure 8: (a) The patch support sections overlaying the tears' regions (red colours), (b) the corresponding Abaqus modelling.

\subsection{Zooming into a region of interest}

As discussed in the introduction, one of the goals of using DIC on historic tapestries is to explore the effects of repairs on the resulting strain field. As such, understanding the effect of patches in mitigating strain localisation is of primary importance. However, when a defect's dimensions (e.g. the length of a tear) is relatively small compared to the size of the entire tapestry, the image resolution of the original photograph and consequently, the detail in the DIC strain measurement is often too low to provide a sufficiently detailed strain map at the length-scale of the tear. In such cases, a natural solution could be to use the camera to zoom into the region around the defect or perhaps to move the camera closer to the tapestry. Part of this investigation explores the merits and effectiveness of such a strategy, in particular we examine how the loss of 
information associated with the zoomed tapestry image affects the uncertainty in the measured strain field [Reu et al. 2015].

To do this, a rectangular region of interest around a tear was scaled up by a factor of three using the MATLAB imresize function, see Figure 9. As before, the strain field generated using FEA (see Section 2.3) was interpolated, mapped, and used to transform both the zoomed speckle pattern and tapestry images. The DIC algorithm was then applied to generate a full-field strain map of the zoomed region. Results are discussed later in Section 3.2.
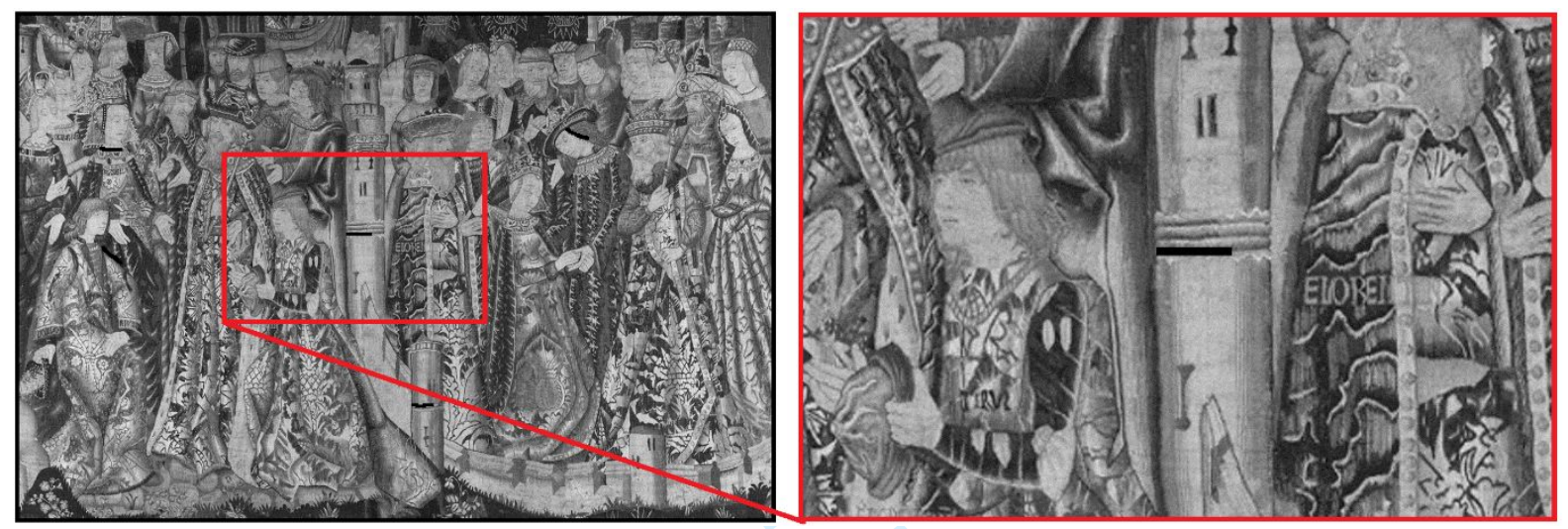

Figure 9: Three times image magnification of the region of interest for the tapestry image containing tears.

\section{Results and discussion}

Four different fabric simulations were conducted, referred to subsequently as Cases 1 to 4 :

1. Homogeneous properties (see Section 2.2.1)

2. Added heterogeneity to Case 1, due to stitched join lines (see Section 2.3.1)

3. Increased heterogeneity of Case 2 by including tears (see Section 2.3.2)

4. Increased heterogeneity of Case 2 by including patches (see Section 2.3.3)

The vertical component of the logarithmic (or Hencky) strain tensor (LE22) is plotted for each type of simulation (Cases 1-4) in Figure 10. The colour legend of the strain map is the same for all images. Sections 3.1-2 examines the use of DIC in measuring the strains across the entire tapestry, as opposed to zooming in to a particular region of interest to examine the strain field near defects such as tears or patches. This latter option will be considered in Section 3.3. 


\subsection{Analysis of entire tapestry}

Figure 10a (Case 1) shows how the strongly non-linear stiffness behaviour fitted in Section 2.2.1 (due to the large degree of crimp in the weft yarns), results in significant LE22 strains at the top of the tapestry (of over $5 \%$ ). The highest strains occur near the top corners of the model due to the combination of self-weight and Poisson's ratio effect. At the bottom of the specimen, as the self-weight loading tends towards zero, so too do the strains, an effect that makes accurate DIC measurement progressively more difficult as the signal to noise ratio approaches zero. Figure 10b (Case 2) includes the heterogeneity caused by the weaker slit stitching. The latter clearly introduce a great deal of complexity into the strain field and result in higher strains towards the top of the tapestry due to the increased compliance caused by the stitched slits. Figure 10c (Case 3) demonstrates that tears in the tapestry create highly localised LE22 strains at the ends of each tear, particularly if a tear is orientated horizontally rather than vertically or diagonally, and serve to decrease the LE22 strains directly above and below the tear. Finally, Figure 10d (Case 4) demonstrates that the use of relatively stiff patches produces lower LE22 strains over and to the sides of the area of the patch but creates slightly higher strains above and below the patch.

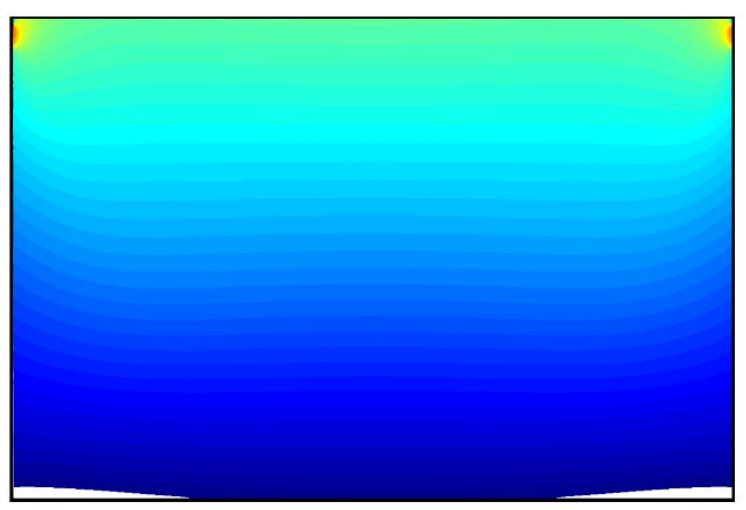

(a)

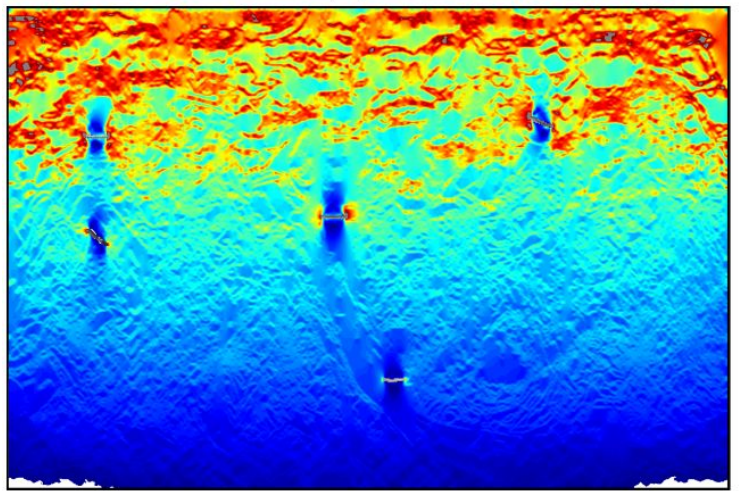

(c)

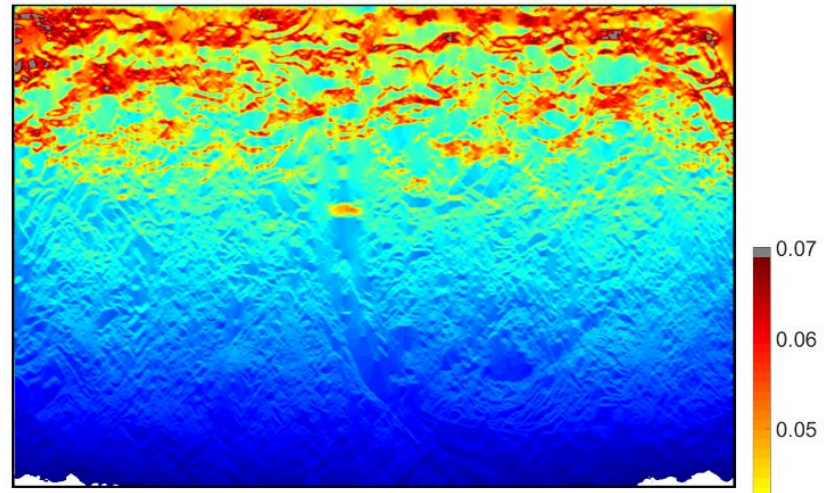

(b)

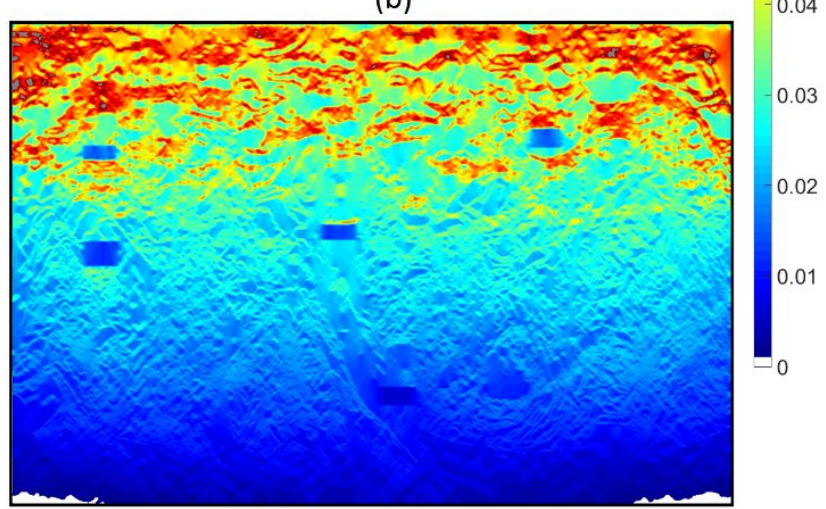

(d) 
Figure 10: Simulated vertical strain fields for the 1.5x1.2m tapestry: (a) with homogenous material assignment, (b) including stitched join lines, (c) including randomised tears along stitched join lines, (d) including patch restorations over tears

These changes are more easily observed by looking at the difference plots of the strain fields. This technique helps to isolate the signal of interest by removing much of the heterogeneous complexity of the tapestry strain field due to stitched slits (noise) as well as the background strain gradient resulting from the self-weight of the tapestry, see Figure 11 (note the change in colour scale compared to Figure 10).

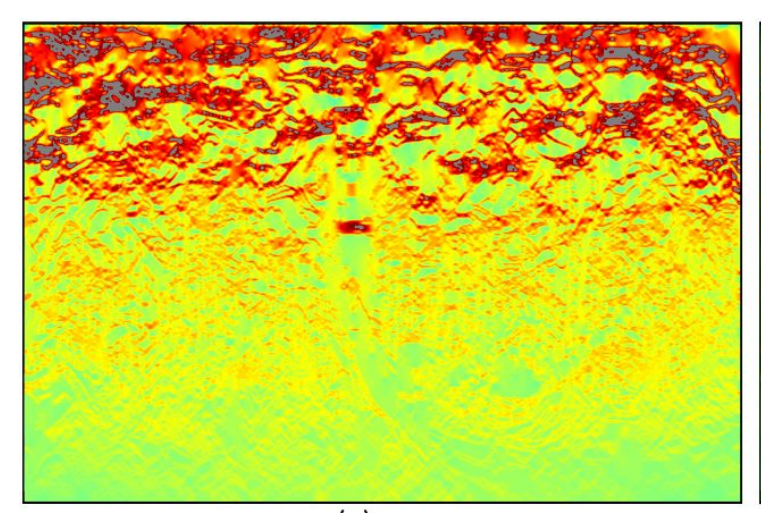

(a)

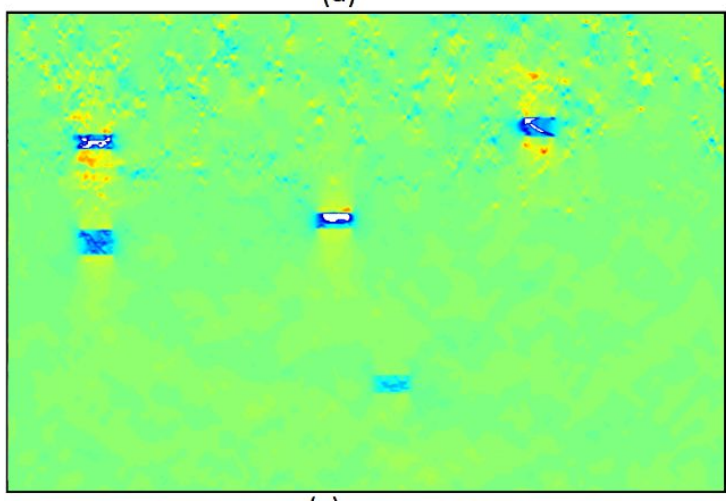

(c)

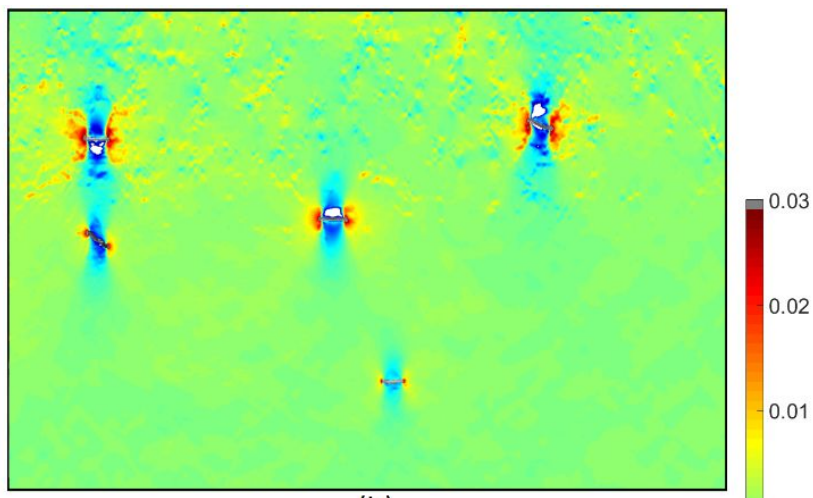

(b)

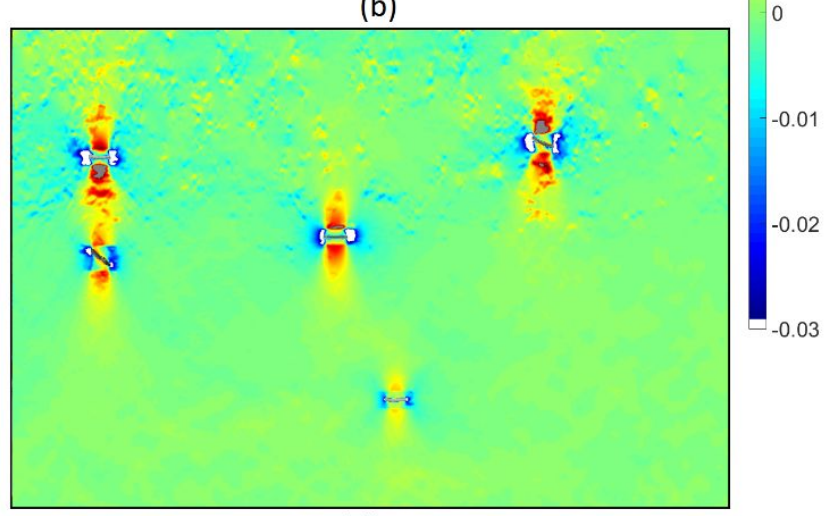

(d)

Figure 11: (a) Homogeneous strain field subtracted from heterogeneous (join line) strain field, (b) heterogeneous strain field subtracted from tear strain field, (c) heterogeneous strain field subtracted from patched strain field, (d) the tear strain field subtracted from the patched strain field.

Figure 11a - the homogeneous strain field (Figure 10a, Case 1) subtracted from the heterogeneous strain field (Figure 10b, Case 2) - shows the increase in LE22 strains due to the stitched slits. Figure $11 \mathrm{~b}$ - the heterogeneous strain field (Fig 10b, Case 2) subtracted from the tear strain field (Figure 10c, Case 3) - highlights the localised increase in strains at the ends of the tears and also reveals a significant relaxation of LE22 strain directly above and below the tears. 
Likewise Figure 11c - the heterogeneous strain field (Figure 10b, Case 2) subtracted from the patched strain field (Figure 10d, Case 4) - clearly highlights a relative reduction in LE22 strain both across and at the sides of the patches but reveals an increase in strain above and below the patches. Finally Figure 11d - the tear strain field (Figure 10c, Case 3) subtracted from the patched strain field (Figure 10d, Case 4) - shows the effect of patching on the torn tapestry strain field. As expected, the patches serve to eliminate the localised stresses previously located at the tips of the tears (shown by a negative change in strain) but do inevitably introduce an increase in strain in the previously unloaded regions above and below the tears.

The strain fields shown in Figure 10 and the resulting changes in the strain fields highlighted in Figure 11 serve as test cases with which to examine the performance of the DIC algorithm using both the speckle pattern image and the inherent tapestry image to track deformations. DIC analysis was performed using correlated solutions VIC-2D (2009) software. The effect of subset size on the accuracy of the DIC measurement was also examined by using three different subset sizes for each case $(33 \times 33,69 \times 69$ and $99 \times 99$ pixels). A step size of 1 pixel was used in all DIC analyses.

\subsection{Evaluation of DIC performance}

Beginning with Case 1, Figure 12 shows that DIC measurements using both the speckle pattern images $(a-c)$ and the inherent tapestry images $(d-f)$ as tracking images provide reasonably accurate results for all subset sizes. The smallest subset size provides greater resolution but also increased error for both tracking images (speckle pattern and tapestry images). 
(a)

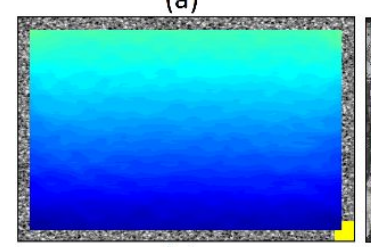

(b)

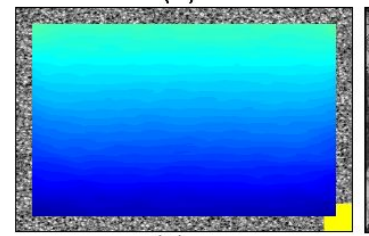

(c)

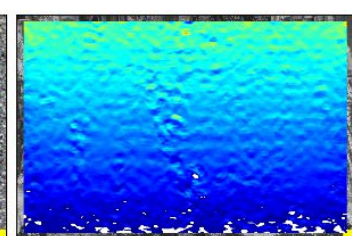

(d)

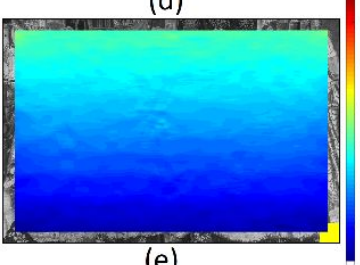

(e)

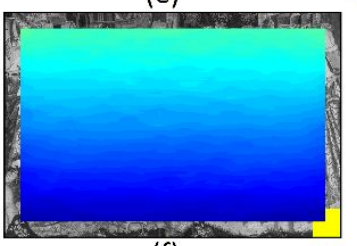

(f)

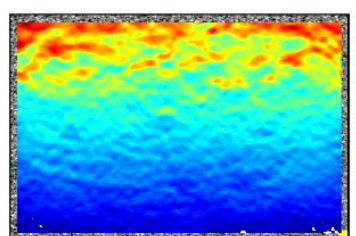

(a)

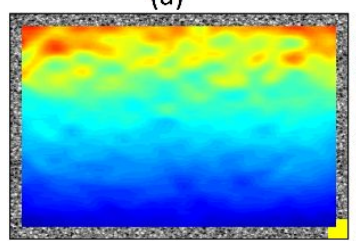

(b)

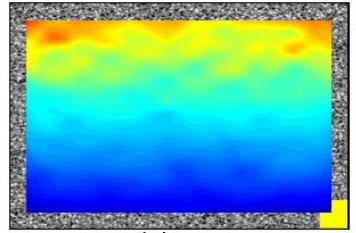

(c)

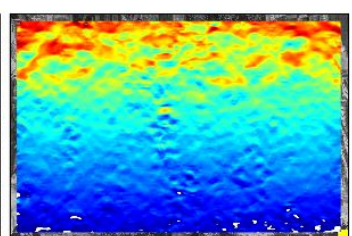

(d)
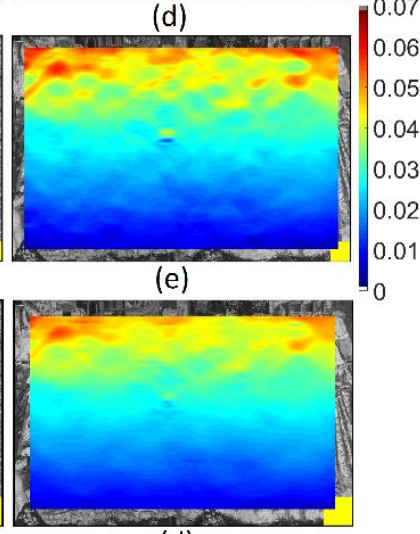

(d)

Figure 12: Left: DIC-measured LE22 strains deformed using predicted simulation strains from homogenous material assignment (Case 1). (a-c) using speckle pattern image to track deformation and subset sizes, shown by yellow corner square, of (a) 33 pixels, (b) 69 pixels, (c) 99 pixels. (d-f) using tapestry image to track deformation and subset sizes of (d) 33 pixels, (e) 69 pixels, (f) 99 pixels.

Figure 13: Right: The DIC-measured LE22 strains deformed using predicted simulation strains from heterogeneous material assignment (Case 2): (a-c) using speckle-pattern images to track deformation and subset sizes shown by yellow corner square, of (a) 33 pixels, (b) 69 pixels, (c) 99 pixels. (d-f) using tapestry image to track deformation and subset sizes of (d) 33 pixels, (e) 69 pixels, (f) 99 pixels

A similar evaluation is conducted for Case 2; a more challenging and complex heterogeneous strain field caused by the stitched slits. Once again, the smaller subset size provides greater resolution (Fig 13a \& 13d) but increased error, whereas the largest subset size decreases resolution (Fig 13c \& 13f) yet decreases error. Both the speckle pattern image and the tapestry image provide similar strain plots, but with slightly more error when using the tapestry image. Case 3 incorporates tears in the tapestry model, creating significant localised strain concentrations. The tears are visible to varying degrees for both the speckle pattern and the tapestry tracking images, though the small hole in the fabric, visible in the simulations has disappeared as a result of the interpolated mapping of the deformation across the tear. Rather than a hole in the fabric, interpolation means that the DIC measures a region of high positive strain. The tears are more visible when located towards the top of the tapestry due to greater loading. 
Once again, the effects of the tears are more clearly seen by creating difference plots, effectively removing the background 'noise' from the signal of interest. As such the DIC result for Case 3 (tapestry with tears, see Figure 14) minus the DIC measurement for Case 2 (heterogeneous tapestry) is presented in Figure 15. Note that similar images created using the speckle pattern rather than the inherent tapestry image are provided in the Appendix (see Figures $A 1$ and A2). Ideally these plots would all be the same as Figure 11b. In practice, the smallest subset size provides the best resolution but at the expense of introducing significant background noise. As might be expected, the result produced when using the speckle pattern image to track deformation is less prone to background noise than that produced when using the inherent tapestry image, though the latter is reasonably successful nonetheless. The larger subset size contains much less background noise but also lacks much of the detail around the tears, the midsubset size is better in this regard but also lacks detail.
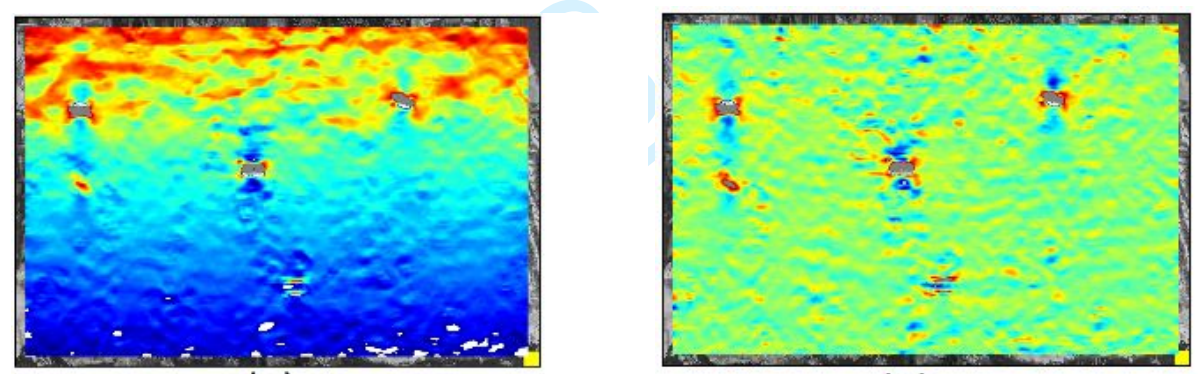

(a)

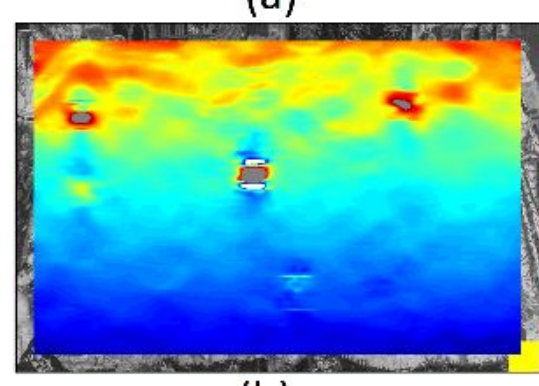

(a)

0.07

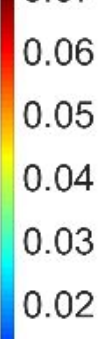

(b)

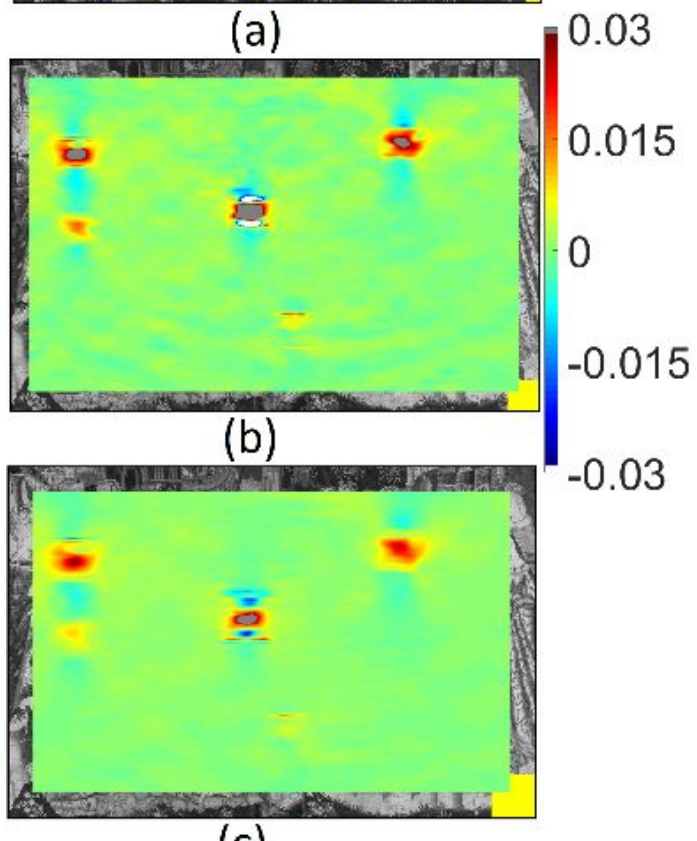

(c)

(c) 
Figure 14: The DIC-measured LE22 strain fields for the images deformed using the computer model with the heterogeneous material assignment and five tears for the tapestry-pattern images by the applied subset sizes of (a) 33 pixels, (b) 69 pixels, (c) 99 pixels.

Figure 15: The difference between the DIC-measured LE22 strain fields for the images deformed using the computer model with the heterogeneous material assignment and five tears and the Figure 13 for the tapestry-pattern images by the applied subset sizes of (a) 33 pixels, (b) 69 pixels, (c) 99 pixels.

Patching the tears decreases the strain concentrations at the end of the tears; nevertheless, depending on the stiffness of the patch, new localised strains emerge above and below the treated areas (see Figures $10 \mathrm{~d}$ and 11c). As before the smallest subset size provides the best resolution and the largest subset size reduces noise at the expense of blurring much of the detail in the strain field.

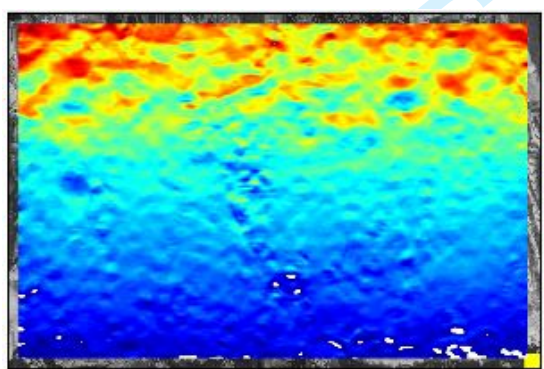

(a)

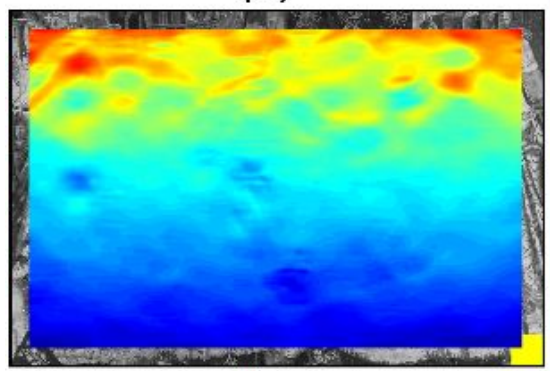

(b)

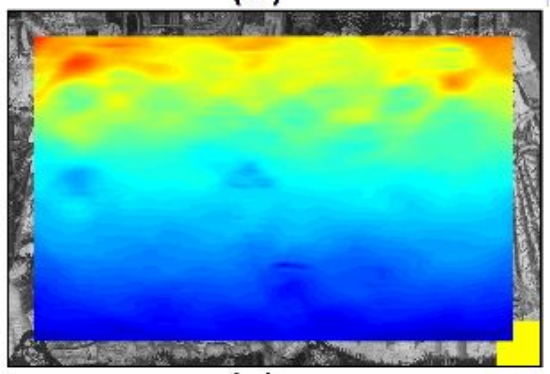

(c)

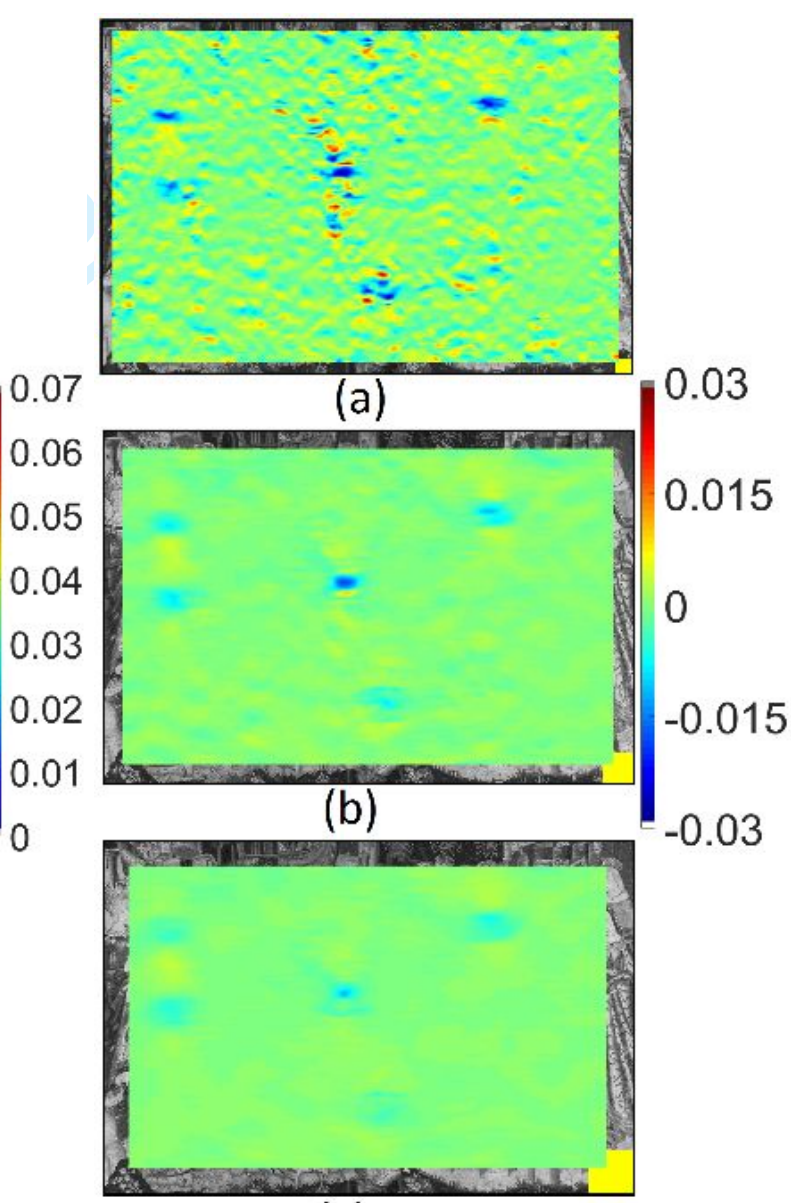

(c) 
Figure 16: The DIC-measured LE22 strain fields for the images deformed using the computer model with the heterogeneous material assignment and five patch support treatments for the tapestry-pattern images by the applied subset sizes of (a) 33 pixels, (b) 69 pixels, (c) 99 pixels.

Figure 17: The difference between the DIC-measured LE22 strain fields for the images deformed using the computer model with the heterogeneous material assignment and five patch support treatments and Figure 12 for the tapestry-pattern images by the applied subset sizes of (a) 33 pixels, (b) 69 pixels, (c) 99 pixels.

Visual assessment of Figures 12 to 17 (and A1-A4 in appendix) suggests that despite the extra noise, the smallest subset size provides the best results for both the speckle pattern and tapestry tracking images. As such the smallest subset size was chosen to create Figure 18, allowing a direct comparison with Figure 11, i.e. comparison between the actual strain difference maps (Figure 11a-d) and the measured strain difference maps produced using the inherent tapestry image and smallest subset size to measure the strains (Figure 18a-d). The results are encouraging, in particular Figure 18d clearly shows the reduction in strain at the end of each of the tears and also the relative increase in strain both above and below the tears due to the bridging effect of the patches (i.e. the unloaded regions originally above and below the tears is now loaded, resulting in an increase in strain). Details around even the lowest tear location are visible, however, significant noise across the maps is also apparent, consequently a priori knowledge of the location of any defects and restoration treatment adds confidence when interpreting the measured strain maps. Attempts to use additional noise filters on the strain maps shown in Figure 18 (e.g. Gaussian etc [MATLAB R2015a, User Manual, Image Acquisition Toolbox]) proved fruitless and generally has a similar effect as increasing the subset size used in the DIC analysis, i.e. a reduction in noise at the cost of decreased resolution. 


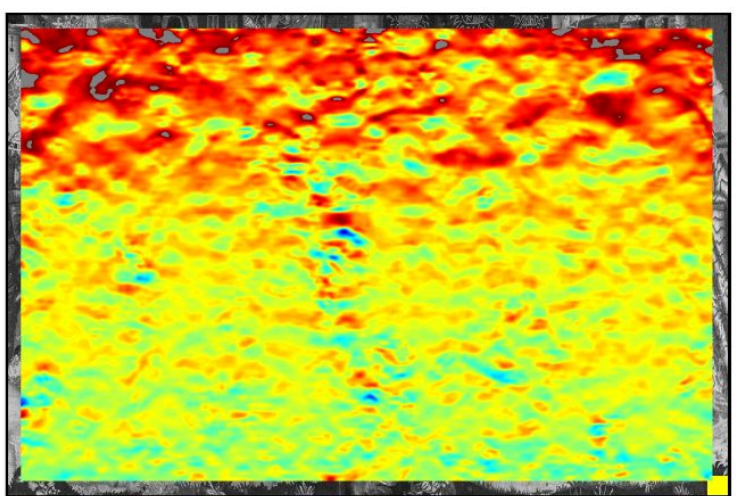

(a)

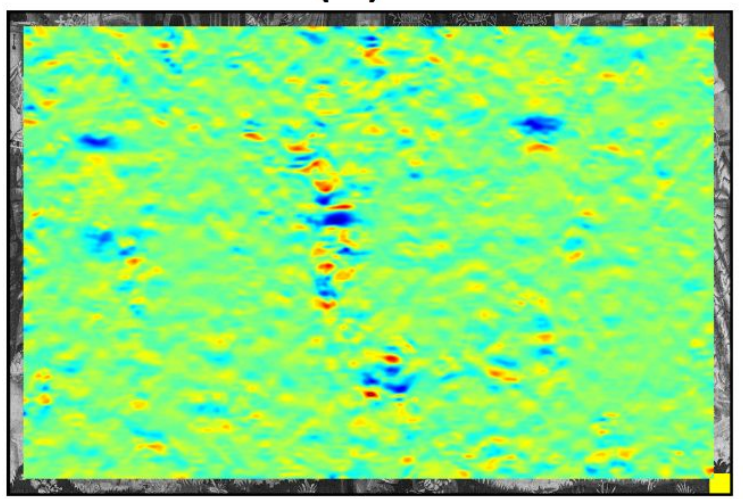

(c)

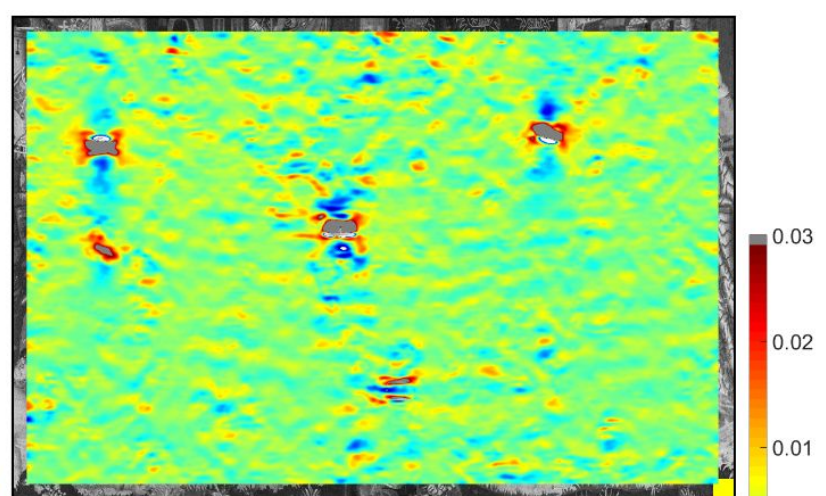

(b)

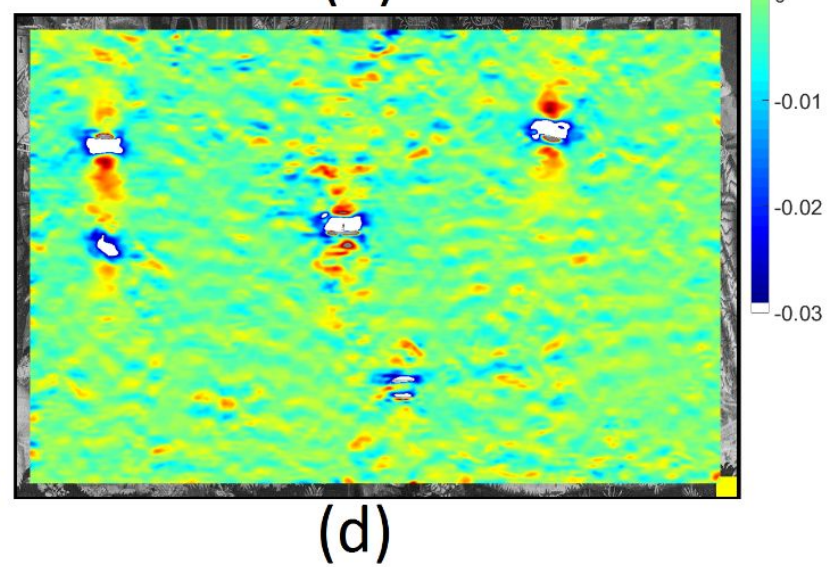

Figure 18: DIC difference plots for the images with the tapestry-patterns and applied subset size of 33 pixels: (a) Homogeneous strain field subtracted from heterogeneous (join line) strain field, (b) heterogeneous strain field subtracted from tear strain field, (c) heterogeneous strain field subtracted from patched strain field, (d) the tear strain field subtracted from the patched strain field.

\subsection{Zooming into a region of interest}

A plausible strategy to more closely examine the strain field around a defect might be to zoom into the region of interest. To investigate the effect of magnification when using the inherent tapestry image to track deformation, a zoomed region of interest containing both stitched join lines and a tear is considered (see Figure 9 and Figure 19). To avoid the material discontinuity associated with the tear (i.e. no material strain actually occurs over the tear), the latter was excluded from the analysis using the 'region of interest' selection tool available in the DIC software (see Figure 20 and A5 in appendix). This strategy eliminates the erroneous positive strain measurement that otherwise occurs across the tears due to interpolation, see for example, Figure 10c. Figures 20a-c and A5a-c illustrate how a smaller subset size allows analysis closer to the tear (because the subsets are not permitted to overlap the hole). Figure 20a-c, shows that use of the speckle pattern to track the deformation leads to much more accurate strain 
measurement compared to that produced using the inherent tapestry image (Figure A5a-c). By zooming into the tapestry image, the amount of information available per subset has been reduced, creating greater error in tracking the displacements (and consequently the strains). This is evident in Figure A5a, created using the tapestry image using the smallest subset size. The same colour legend as that used in Figure 10 is employed but now the DIC strain measurement contains considerably more noise, making the original strain field barely recognisable. The speckle pattern copes much better with the magnification. As usual, increasing the subset size reduces the measurement error for both speckle pattern and inherent tapestry tracking images at the cost of losing resolution. This example demonstrates an inevitable constraint when using a tapestry image to visualise the strain field with DIC. Images with less complexity and detail require larger subset sizes to reduce measurement error to acceptable levels but in doing so, reduce the resolution of the measured strain map.

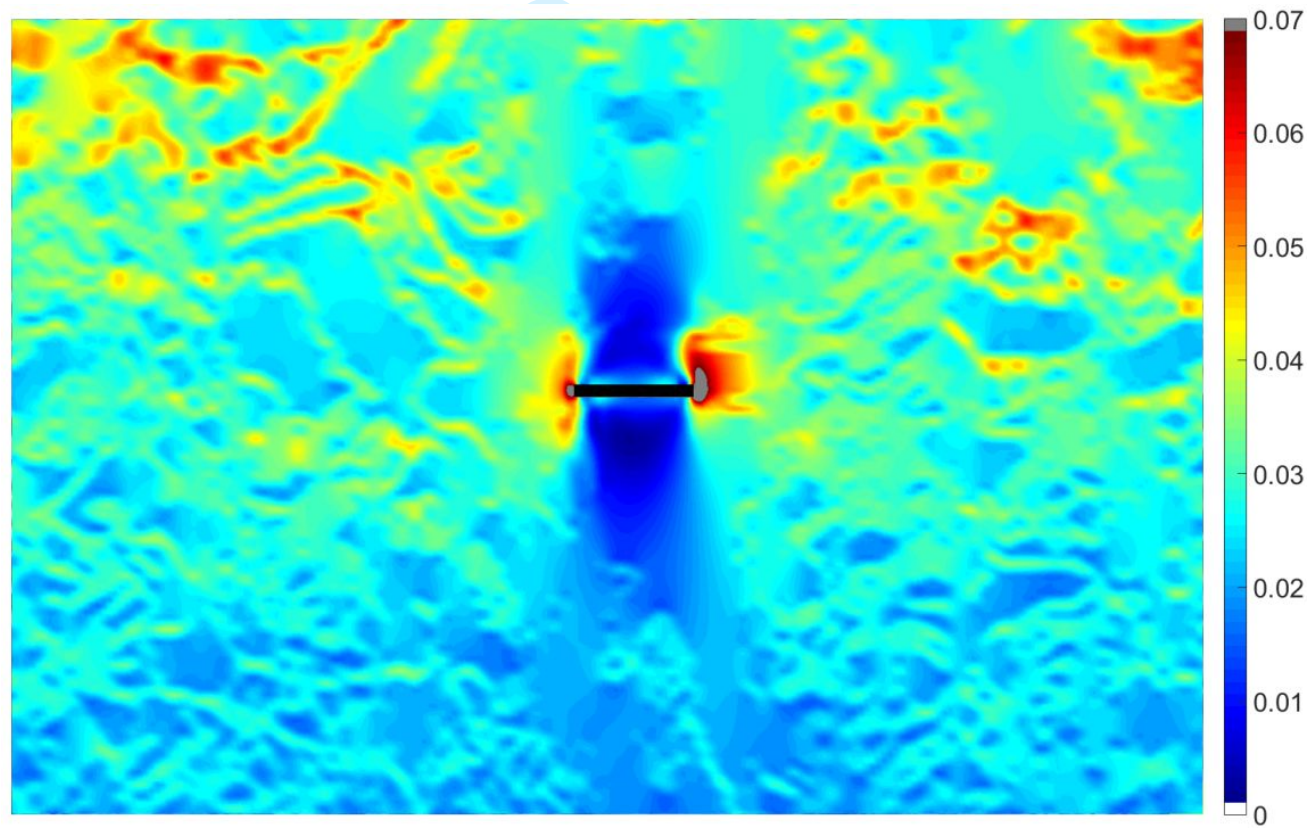

Figure 19: The Abaqus LE22 strain contours for the selected region of interest

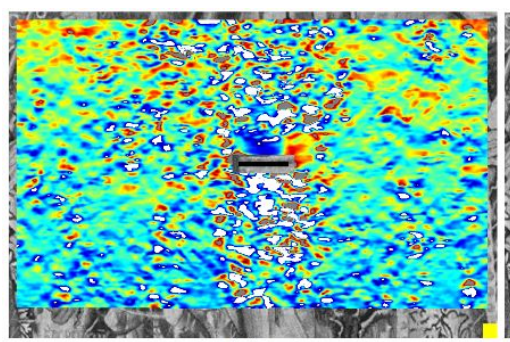

(a)

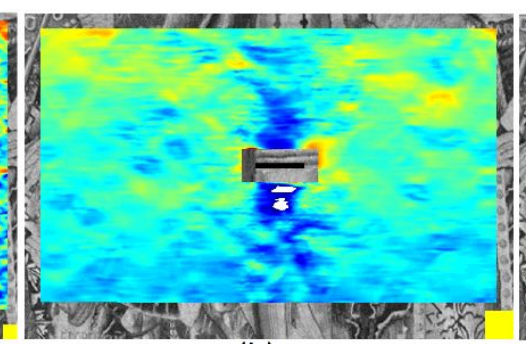

(b)

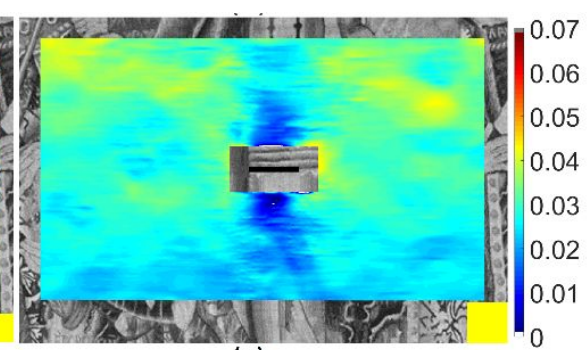

(c) 
Figure 20: The DIC-measured LE22 strain fields for the magnified deformed images using the tapestry-pattern images by the applied subset sizes of (a) 33 pixels, (b) 69 pixels, (c) 99 pixels.

\section{Conclusion}

A method to evaluate the potential of DIC in measuring the full-field strains across historical tapestries, prior to potentially time-consuming and costly experimental investigation, has been demonstrated. The technique involves generating a reasonably realistic, tapestry-specific strain field by projecting the image dependent heterogeneity of the tapestry onto a finite element mesh. The strain field generated by the simulation is then used as a test case to evaluate the measurement error produced using DIC with the inherent tapestry image to track deformations.

Use of both speckle-patterns and tapestry images throughout the investigation guarantees that any difference between the two sets of results is a consequence of the tracking image rather than from interpolation error introduced when using the simulated strain field to deform the pixilated images analysed using the DIC algorithm. Given the interest of conservators in understanding the effect of restoration treatments on the subsequent strain field in a tapestry (and consequently its propensity for subsequent damage), the effect of tears and patches was superposed onto the already complex strain field associated with self-weight loading, non-linear stiffness and the heterogeneous stitched nature of the tapestry.

An effective tool in highlighting the changes induced by such treatments was the use of difference maps. The latter allows much of the complexity of the strain field to be eliminated in order to focus on the effects of conservation. The results of this particular analysis have been encouraging; compare Figures 11 and 18 (and A1-A4 in appendix). However, it should be noted that all decisions in conducting this investigation have been favourable to producing good results. For example:

- the chosen tapestry image contains considerable detail and complexity

- the tapestry is reasonably large

- the material behaviour used in the simulations is relatively compliant and the areal density relatively high

- the tears introduced into the analysis are relatively large 
- errors due to out-of-plane displacements of the tapestry have been neglected (the significance of this error will depend on whether 2-D or 3-D DIC is conducted)

For this reason, the results shown in this investigation are likely to lie towards the more successful end of the spectrum of results that might be expected when conducting DIC on historic tapestries. No doubt, other choices could produce less fruitful analysis. However, the main aim of the paper is to demonstrate a strategy for bespoke pre-experimental assessment of a given tapestry prior to conducting actual experiments. In some sense the technique serves as a tool for management of expectations.

The investigation highlights the issue that the optimum subset size is not well-defined and depends to a large extent on the user's preference - how much measurement accuracy can be sacrificed for the sake of increased resolution and which region of the tapestry is to be analysed. The technique of generating difference maps proved very useful in highlighting the features of interest (i.e. the effect of patching tears). Such maps are relatively easy to produce using computational analysis but more difficult to obtain in practice. To do so would require the tapestry to be hung in an almost identical way before and after conservation to create equivalent difference maps from experimental data. This conclusion provides inspiration for the development of experimental equipment that can facilitate this endeavour.

\section{Acknowledgement:}

The authors wish to thank the Leverhulme Trust for the grant 'From the Golden Age to the Digital Age: Modelling and Monitoring Historic Tapestries', (Ref: RPG-2015-179). Also, the authors would like to thank the staff of Glasgow Museums for their continuous support without which it would not have been possible to conduct this study.

\section{References:}

ABAQUS (2018), ABAQUS 6.14-2 User's Manual. Online Documentation: Dassault Systèmes AMIOT, F., BORNERT, M., DOUMALIN, P., DUPRE, J. C., FAZZINI, M., ORTEU, J. J., POILANE, C., ROBERT, L., ROTINAT, R., TOUSSAINT, E., WATTRISSE, B. \& WIENIN, J. S. 2013. Assessment of Digital Image Correlation Measurement Accuracy in the Ultimate Error Regime: Main Results of a Collaborative Benchmark. Strain, 49, 483-496. 
BENSON. S. J., LENNARD. F., SMITH. M. J., Like-with-like: a comparison of natural and synthetic stitching threads used in textile conservation, ICOM-CC, $17^{\text {th }}$ Triennial conference, Melbourne (2014)

BORNERT, M., DOUMALIN, P., DUPRE, J. C., POILANE, C., ROBERT, L., TOUSSAINT, E. \& WATTRISSE, B. 2012. Short Remarks About Synthetic Image Generation in the Context of the Assessment of Sub-Pixel Accuracy of Digital Image Correlation. Icem15: 15th International Conference on Experimental Mechanics.

CANNY, JOHN. "A Computational Approach to Edge Detection," IEEE Transactions on Pattern Analysis and Machine Intelligence, Vol. PAMI-8, No. 6, 1986, pp. 679-698.

DUREISSEIX, D., COLMARS, J., BALDIT, A., MORESTIN, F. \& MAIGRE, H. 2011. Follow-up of a panel restoration procedure through image correlation and finite element modeling. International Journal of Solids and Structures, 48, 1024-1033.

HARRISON, P., ALVAREZ, M. F. \& ANDERSON, D. 2017. Towards comprehensive characterisation and modelling of the forming and wrinkling mechanics of engineering fabrics. International Journal of Solids and Structures.

HU, J., 2004. Structure and Mechanics of Woven Fabrics, 1st ed. Woodhead Publishing Limited, Cambridge. doi: 10.1017/CBO9781107415324.004

GAUVIN C., JULLIEN D., DOUMALIN P., DUPRÉ J.-C., and GRIL J. (2014) Image Correlation to Evaluate the Influence of Hygrothermal Loading on Wood, Strain, 50, 428-435

KHENNOUF, D., DULIEU-BARTON, J. M., CHAMBERS, A. R., LENNARD, F. J. \& EASTOP, D. D. 2010. Assessing the Feasibility of Monitoring Strain in Historical Tapestries Using Digital Image Correlation. Strain, 46, 19-32.

KE, X. D., SCHREIER, H. W., SUTTON, M. A. \& WANG, Y. Q. 2011. Error Assessment in Stereo-based Deformation Measurements Part II: Experimental Validation of Uncertainty and Bias Estimates. Experimental Mechanics, 51, 423-441.

LAVA, P., COOREMAN, S., COPPIETERS, S., DE STRYCKER, M. \& DEBRUYNE, D. 2009. Assessment of measuring errors in DIC using deformation fields generated by plastic FEA. Optics and Lasers in Engineering, 47, 747-753.

LAVA, P., COOREMAN, S. \& DEBRUYNE, D. 2010. Study of systematic errors in strain fields obtained via DIC using heterogeneous deformation generated by plastic FEA. Optics and Lasers in Engineering, 48, 457-468.

LECOMPTE, D., SMITS, A., BOSSUYT, S., SOL, H., VANTOMME, J., VAN HEMELRIJCK, D. \& HABRAKEN, A. M. 2006. Quality assessment of speckle patterns for digital image correlation. Optics and Lasers in Engineering, 44, 1132-1145.

LENNARD, F. AND HAYWARD, M. (Eds.) (2006) Tapestry Conservation: Principles and Practice. Series: Series in Conservation and Museology. Butterworth-Heinemann: Oxford. ISBN 9780750661843

LENNARD, F. AND DULIEU-BARTON, J. M., (2014) Quantifying and visualizing change: Strain monitoring of tapestries with digital image correlation, Studies in Conservation, 59:4, 241-255

LITHGOW, K., The Icon Conference, Positive Futures, April 2013 
LUO, P. F., CHAO, Y. J., SUTTON, M. A. \& PETERS, W. H. 1993. Accurate Measurement of 3-Dimensional Deformations in Deformable and Rigid Bodies Using Computer Vision. Experimental Mechanics, 33, 123132.

MATLAB, MathWorks ${ }^{\circledR}$, Online Documentation

ORTEU, J. J. 2009. 3-D computer vision in experimental mechanics. Optics and Lasers in Engineering, 47, 282-291.

ORTEU, J. J., GARCIA, D., ROBERT, L. \& BUGARIN, F. 2006. A speckle-texture image generator. SPECKLE06: Speckles, From Grains to Flowers, 6341, U110-U115.

PAN, B., XIE, H. M., WANG, Z. Y., QIAN, K. M. \& WANG, Z. Y. 2008. Study on subset size selection in digital image correlation for speckle patterns. Optics Express, 16, 7037-7048.

PAN, B., 2013. Bias error reduction of digital image correlation using Gaussian pre-filtering. Optics and Lasers in Engineering, 51(10): 1162-1167

PAN, B., 2018. Digital image correlation for surface deformation measurements: historical developments, recent advances and future goals. Measurement Science and Technology, 29(8): 082001

REU, P. L., SWEATT, W., MILLER, T. \& FLEMING, D. 2015. Camera System Resolution and its Influence on Digital Image Correlation. Experimental Mechanics, 55, 9-25.

SCHREIER, H. W., BRAASCH, J. R. \& SUTTON, M. A. 2000. Systematic errors in digital image correlation caused by intensity interpolation. Optical Engineering, 39, 2915-2921.

SUTTON, M. A., Orteu, J. J., Schreier, H. 2009. Image correlation for shape, motion and deformation measurements. Basic concepts, theory and applications. Springer

SUTTON, M. A., YAN, J. H., TIWARI, V., SCHREIER, H. W. \& ORTEU, J. J. 2008. The effect of out-of-plane motion on 2D and 3D digital image correlation measurements. Optics and Lasers in Engineering, 46, 746757.

TANG, Z. Z., LIANG, J., XIAO, Z. Z., GUO, C. \& HU, H. 2010. Three-dimensional digital image correlation system for deformation measurement in experimental mechanics. Optical Engineering, 49.

WANG, Y., LAVA, P., COPPIETERS, S., DE STRYCKER, M., VAN HOUTTE, P. \& DEBRUYNE, D. 2012.

Investigation of the Uncertainty of DIC Under Heterogeneous Strain States with Numerical Tests. Strain, 48, 453-462. 